\title{
Liver Transplantation during Fifty-Three Years' Experience with Randomized Controlled Trials of Emergency Portacaval Shunt for Bleeding Esophageal Varices in Cirrhosis
}

\section{Marshall J Orloff*}

Department of Surgery, School of Medicine, University of California, San Diego, California, USA

\section{Abstract}

Background: Currently, emergency treatment of Bleeding Esophageal Varices (BEV) consists mainly of endoscopic and pharmacologic measures, with use of TIPS when bleeding is not controlled. Surgical shunt has been relegated to salvage of survivors of failed endoscopic, pharmacologic, and TIPS treatment. We have conducted 10 prospective studies of Emergency Porta Caval Shunt (EPCS) during 46 years.

BEV in cirrhosis has been considered an indication for Liver Transplantation (LT) by some respected LT programs. This important issue was examined in our two recent Randomized Controlled Trials (RCTs) in 365 unselected, consecutive patients with acute BEV conducted from 1988 to 2011.

Study design: In RCT No. 1, 211 unselected, consecutive cirrhotic patients with acute BEV ("all comers") were randomized to Emergency Endoscopic Sclerotherapy (EEST) ( $n=106)$ or EPCS ( $n=105)$. In RCT No. 2, 154 unselected consecutive cirrhotic patients with acute BEV ("all comers") were randomized to TIPS ( $n=78)$ or EPCS ( $n=76$ ). In each RCT, the two treatment groups were compared with regard to effect on survival, control of bleeding, encephalopathy, and direct cost of care. Diagnostic workup was completed within $6 \mathrm{hr}$ and primary treatment was initiated within 8 to 12 hours. Regular follow-up was accomplished in $100 \%$ of patients. In RCT No. 1, 96\% of patients underwent more than 10 years of follow-up, or until death. In RCT No. 2, follow-up lasted for 5 to 10 years in $85 \%$, and 3 to 4.5 years in the remainder. Patients were evaluated for LT on admission and at regular intervals thereafter. In addition to the two RCTs, the analysis of LT was supplemented in 1300 unrandomized cirrhotic patients who previously underwent Porta Caval Shunt (PCS) with 100\% follow-up.

Results: Clinical characteristics of each group were similar at the time of enrollment. The distribution of patients in Child risk classes was almost identical in the two groups in each RCT. Almost one-third of patients in each group were in Child class C. Permanent control of bleeding was achieved by sclerotherapy in only $20 \%$ and by TIPS in only $22 \%$. In contrast, EPCS permanently controlled bleeding in $97 \%$ to $100 \%(p<0.001)$. Sclerotherapy and TIPS patients required twice as many units of blood transfusion as EPCS patients. Survival rate at all time intervals and in all Child classes was significantly greater following EPCS than after sclerotherapy and TIPS $(p<0.001)$. Median survival was over 10 years following EPCS, compared to 1.99 years following TIPS. EPCS remained permanently patent in $97 \%$ of patients. In contrast, stenosis or occlusion of TIPS was demonstrated in $84 \%$ of patients, $63 \%$ of whom underwent TIPS revision, which failed in $80 \%$. Recurrent episodes of encephalopathy developed in $35 \%$ of sclerotherapy patients and $61 \%$ of TIPS patients. In contrast, encephalopathy occurred in $15 \%$ of EPCS patients in RCT No. 1 and $21 \%$ of EPCS patients in RCT No. 2. Direct costs of care, measured as charges per year, were 5 to 7 times greater in the sclerotherapy group $(\$ 168,100)$ and TIPS groups $(\$ 264,800)$ than in the EPCS group $(\$ 39,000)(p<0.001)$

Of the 365 randomized patients in the two RCTs, only $23(6.3 \%)$ were ultimately referred for LT, mainly because of progressive liver failure. Of these, only $11(3 \%)$ were approved for LT, and only $8(2.2 \%)$ underwent LT. One- and 5 -year LT survival rates were $0.68 \%$ and 0 , compared to $81 \%$ and $73 \%$ after EPCS. In the 1300 unrandomized PCS patients, $50(3.8 \%)$ were referred and $19(1.5 \%)$ underwent LT. Five-year survival rate was $53 \%$ compared to $72 \%$ for all 1300 patients.

Conclusions: EPCS was uniformly effective in treatment of BEV, while sclerotherapy and TIPS were disappointing. EPCS permanently stopped variceal bleeding, almost never became occluded, accomplished long-term survival that was more than 5 times survival rate following sclerotherapy or TIPS, and was much less costly than sclerotherapy or TIPS. If bleeding is permanently controlled, as occurred invariably following EPCS, cirrhotic patients with BEV seldom require LT. PCS is effective first-line and long-term treatment. Should LT be required in patients with PCS, although technically more demanding, numerous studies have shown that PCS does not increase mortality or complications. Neither EST nor TIPS are effective emergency or long-term therapeutic measures.

\begin{abstract}
Abbreviations: LT: Liver Transplantation; BEV: Bleeding Esophageal Varices; TIPS: Transjugular Intrahepatic Portosystemic Shunt; EPCS: Emergency Portacaval Shunt; PSE: Portal Systemic Encephalopathy; RCT: Randomized Controlled Trial; UCSD: University of California, San Diego; UGI: Upper Gastrointestinal; ICU: Intensive Care Unit; PCS: Portacaval Shunt; EGD: Esophagogastroduodenoscopy; EEST: Emergency Endoscopic Sclerotherapy; HV: Hepatic Vein; IVC: Portal Vein; PV: Portal Vein; PRBC: Packed Red Blood Cells; PTFE: Polytetrafluorethylene; HGPCS: Prosthetic H-Graft Portacaval Shunt; DSRS: Distal Splenorenal Shunt
\end{abstract}

*Corresponding author: Marshall J. Orloff, Department of Surgery, School of Medicine, University of California, San Diego, California, USA, E-mail: morloff@ ucsd.edu

Received March 26, 2013; Accepted July 29, 2013; Published August 06, 2013

Citation: Orloff MJ (2013) Liver Transplantation during Fifty-Three Years' Experience with Randomized Controlled Trials of Emergency Portacaval Shunt for Bleeding Esophageal Varices in Cirrhosis. Surgery Curr Res 3: 140. doi:10.4172/2161-1076.1000140

Copyright: (C) 2013 Orloff MJ. This is an open-access article distributed under the terms of the Creative Commons Attribution License, which permits unrestricted use, distribution, and reproduction in any medium, provided the original author and source are credited. 


\section{Introduction}

Bleeding Esophageal Varices (BEV) is a common and highly lethal complication of cirrhosis. Numerous studies have shown that BEV is responsible for much of the high mortality rate associated with cirrhosis $[1,2]$. If the varices remain untreated after recovery from a bout of acute bleeding, we [3] and others [4] observed a 95\% incidence of recurrent bleeding, and death within 2 to 5 years in $90 \%$ to $100 \%$ of the patients.

A number of modalities are used today for treatment of acute $\mathrm{BEV}$, including endoscopic variceal ligation, endoscopic sclerotherapy, pharmacologic measures and, if these fail to obtain immediate and prolonged control of bleeding, Transjugular Intrahepatic Portosystemic Shunt (TIPS). Surgical Portal-Systemic Shunt (PSS) is infrequently used today, in large part because of the belief, unsubstantiated by prospective randomized controlled trials involving unselected patients, that surgical shunts cause frequent Portal-Systemic Encephalopathy (PSE) and liver failure. Consequently, surgical PSS has been relegated to the seldom used salvage of survivors of failed endoscopic, pharmacologic, and TIPS treatment.

Liver Transplant (LT) is the only curative treatment of cirrhosis. Some respected transplant centers have considered BEV in cirrhosis to be an indication for LT. It has been proposed that LT be considered the treatment of choice for "patients with advanced liver disease after failure of sclerotherapy" [5,6] and for "all patients with end-stage liver disease (group C) and variceal bleeding... in the absence of any contraindications" [5-7]. Regrettably, there have been no randomized controlled trials of LT following any of the emergency modalities of therapy for BEV to support or contradict these proposals. All reported data on LT in patients with BEV have been based on retrospective reviews of medical records. Furthermore, recommendations regarding LT in patients with BEV generally have not taken into account the well known limitations, particularly insufficient donor organs.

During the 53 years from 1958 to 2011 we performed 10 prospective studies of EPCS [8-17]. A total of 956 patients were involved in these studies of acute BEV, which is the largest reported experience with EPCS in the world. The unique features of our studies that, together, make them different from other reported investigations are as follows: (1) EPCS was undertaken within $20 \mathrm{hrs}$ of initial contact of the patient with our institution, usually within $8 \mathrm{hrs}$; (2) the patients were unselected and consecutive, which means that all patients with bleeding varices ("all comers") regardless of their condition, were entered in the studies and treated; (3) the studies were prospective, which means that the patients were managed according to a well-defined and consistently applied protocol, and specific data were collected at the time of diagnosis, treatment, and follow-up; and (4) the patients were followed up monthly for the first year and every 3 months thereafter for life, such that the 1-, 5-, and 10-years follow-up rates were 100, 98, and 97\%, respectively.

Our most recent studies of EPCS have been two consecutive Randomized Controlled Trials (RCTs) in unselected, consecutive patients with acute BEV. The first of these RCTs (RCT No. 1) compared Emergency Endoscopic Sclero Therapy (EEST) versus EPCS in 211 unselected consecutive patients from April 8, 1988, until December 31, 2005. It was known as the San Diego Bleeding Esophageal Varices Study $[15,16]$. The second RCT (RCT No. 2) compared TIPS versus EPCS in 154 unselected consecutive patients with acute BEV [17]. It was undertaken after entry in the first RCT had ended and was separate and distinct from the first RCT. It was conducted from July 25, 1996, until July 31, 2011. These two RCTs will be discussed in detail in this report.

In both of our RCTs, beginning with the index admission and regularly thereafter, all patients were evaluated by the UCSD liver transplantation program for indications for LT. If and when patients exhibited progressive liver failure, they underwent extensive evaluation for LT. As part of our analysis, we examined the question of the need for LT after the life-threatening problem of BEV had been addressed [18]. In addition, the effect of EST or EPCS on the conduct and outcome of LT was examined.
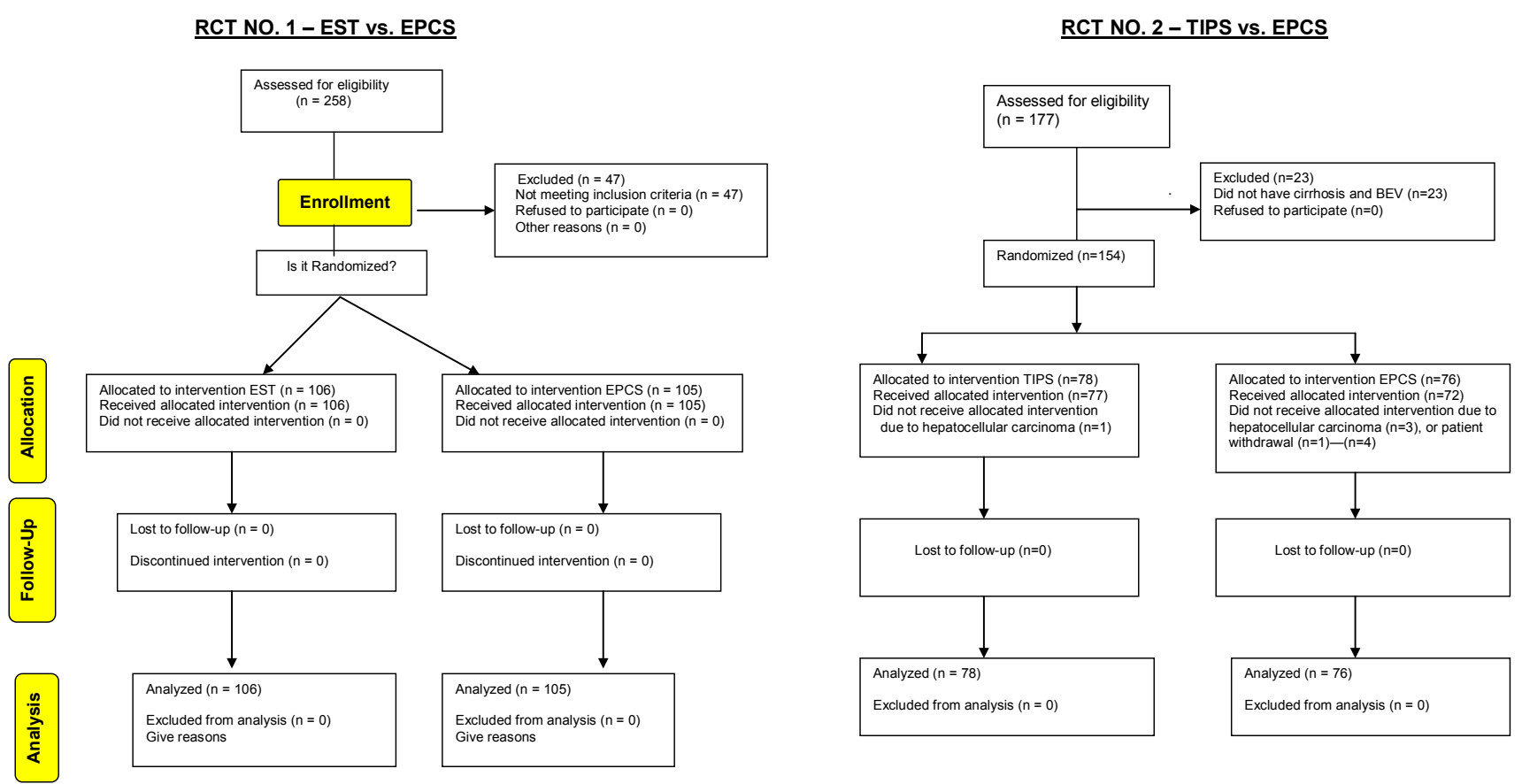

Figure 1: Consort flow diagrams showing the overall design and conduct of the two prospective randomized controlled trials (RCTs) [21-23]. 
As a supplement to the RCT data, we have analyzed our results regarding LT in 1300 unrandomized referred patients in whom we performed Porta Caval Shunt (PCS) beginning in 1978, 600 as an emergency PCS and 700 electively.

\section{Patients and Methods}

\section{Design of RCTs No. 1 and No. 2}

Figure 1 is a Consort Flow Diagram that shows the overall design and conduct of the two RCTs [19-21]. In unselected, consecutive patients ("all comers") who entered UCSD Medical Center with acute $\mathrm{BEV}$, the objectives were to compare Emergency Direct Porta Caval Shunt (EPCS) versus EEST in RCT No. 1 and versus TIPS in RCT No. 2 with regard to influence on survival, control of bleeding, PSE, and economic costs. Ninety-one board-certified gastro $\neg$ enterologists, emergency physicians, and surgeons in the counties of San Diego, Imperial, Orange, and Riverside, with a population of 8.5 millions, agreed to promptly refer all of their patients with acute BEV to UCSD Medical Center for entry into the RCT. Patient entry in RCT No. 1 lasted from April 8, 1988, until July 25, 1996, and follow-up continued until December 31, 2005, 17 and $3 / 4$ years after the start of the study. Patient entry in RCT No. 2 lasted from July 25, 1996, until October 8, 2003, and follow-up continued until July 31, 2011, 15 years after the start of the study.

Our recent publications [15,17] described our RCTs and provided full information on the protocols and methods. These include (1) design of study; (2) patient eligibility; (3) definitions (bleeding esophageal varices (BEV), unselected consecutive patients ("all comers"), Emergency Endoscopic Sclerotherapy (EEST), long-term Endoscopic Sclera Therapy (EST), Emergency Porta Caval Shunt (EPCS), TIPS, failure of emergency primary therapy, failure of long-term therapy, rescue therapy, informed consent; (4) randomization; (5) diagnostic work-up; (6) quantitative Child's classification; (7) initial emergency therapy during work-up; (8) Emergency Endoscopic Sclero Therapy (EEST); (9) Emergency Portacaval Shunt (EPCS); (10) TIPS; (11) posttreatment therapy; (12) lifelong follow-up; (13) quantitation of PortalSystemic Encephalopathy (PSE); (14) data collection; (15) direct costs of care; (16) TIPS-specific follow-up.

\section{Eligibility}

All patients without selection with Upper Gastrointestinal (UGI) bleeding, defined as blood in the esophagus, stomach or duodenum, who entered the UCSD emergency department directly, or were transferred to UCSD from an area hospital, or developed bleeding while hospitalized in UCSD Medical Center were eligible for investigation. Such patients were enrolled in the RCT if they met the following 5 conditions: (1) requirement for two or more units of blood transfusion, (2) clinical and laboratory findings of cirrhosis, (3) upper endoscopy that showed esophageal varices, (4) no other lesion that could reasonably account for bleeding of the observed magnitude, and (5) UGI bleeding that had been observed within $48 \mathrm{hr}$ of study entry and, therefore, was considered acute bleeding.

\section{Informed consent}

Informed consent was obtained by a physician co-investigator, from every patient before randomization to the treatment groups, and was witnessed by a third party who was not involved in the study. Consent was obtained after the patient was given a thorough explanation of the study, including the risks and benefits of all treatment options, including those not involved in the RCT. The procedure for obtaining consent and the consent form were regularly reviewed and approved by the Human Subjects Committee (Institutional Review Board) and the National Institutes of Health (NIH).

\section{Randomization}

After emergency endoscopy demonstrated esophageal varices and no other lesion that could reasonably account for the bleeding, the diagnostic workup provided clear evidence of cirrhosis of the liver, and informed consent was obtained, the patients were randomized by drawing a card from an opaque sealed envelope, to either an EEST group or an EPCS group in RCT No. 1, or to either TIPS or EPCS in RCT No. 2. Sealed designation cards were prepared by a statistician according to a computer-generated block randomization design, without the knowledge of the physicians participating in the study.

\section{Direct cost of care}

There have been no reports of the costs of any of the widely used forms of emergency treatment of BEV. In each RCT, the two groups were compared with regard to direct costs of care [16]. Complete UCSD charges were obtained for every patient entered in each RCT continuously for more than 10 years. In addition, all referring hospitals and referring physicians signed agreements to provide complete records of charges as they occurred. Prior to initiation of the study, UCSD Medical Center agreed to promptly provide copies of all hospital and outpatient charges on all patients at the time when the patients and insurance carriers were billed. Similarly, the UCSD Medical Group, which does the professional fee billing for all physicians who care for patients at UCSD Medical Center, agreed to promptly provide copies of all professional fee bills.

\section{Unrandomized portacaval shunt patients}

Beginning in 1978 when LT became available to us, we performed Porta Caval Shunt (PCS) for BEV in 1300 referred cirrhotic patients at UCSD Medical Center Hospitals and the San Diego Veterans Administration Hospital. Patients were studied prospectively according to a well-defined protocol with on-line data collection to determine the indications for subsequent LT. The diagnostic work-up was identical in the unrandomized patients and the RCT patients. From 1978 until the start of our RCT in 1988, EPCS was performed for acute variceal bleeding within 8 hours of initial contact in 600 unselected patients, all comers included. Elective PCS was done over a period of 20 years in 700 selected patients referred to us only after recovering from one or more bouts of BEV elsewhere. Follow-up rate was $100 \%$ at 5 years and $97 \%$ at 10 years.

\section{Statistical analysis}

The survival rates in the EST and EPCS groups in RCT No. 1 and in the TIPS and EPCS groups in RCT No. 2, and in the subgroups determined by the Child's risk class were calculated and plotted using the Kaplan-Meier method, and compared using the log-rank test. Statistical comparisons between the EST and EPCS groups in RCT No. 1 and between the TIPS and EPCS groups in RCT No. 2 were performed as follows: The proportion of subjects referred for LT evaluation, the proportion approved for LT, and the diagnosis of the LT candidates, were compared using Fisher's Exact Test (FET). The time after entry in the RCT to LT evaluation and the duration of survival after evaluation and since entry were compared using the log-rank test. The MELD at time of LT was evaluated using the Wilcoxon rank test and the Child's risk class at LT evaluation using Fisher's exact test. Statistical significance was considered at the 0.05 level. 
Citation: Orloff MJ (2013) Liver Transplantation during Fifty-Three Years' Experience with Randomized Controlled Trials of Emergency Portacaval Shunt for Bleeding Esophageal Varices in Cirrhosis. Surgery Curr Res 3: 140. doi:10.4172/2161-1076.1000140

Page 4 of 13

\begin{tabular}{|c|c|c|c|c|c|c|}
\hline \multirow[b]{2}{*}{ Characteristic } & \multicolumn{3}{|l|}{ RCT No. 1} & \multicolumn{3}{|l|}{ RCT No. 2} \\
\hline & $\begin{array}{l}\text { EST } \\
(n=106)\end{array}$ & $\begin{array}{l}\text { EPCS } \\
(n=105)\end{array}$ & $\begin{array}{l}\mathrm{P} \\
\text { Value }\end{array}$ & $\begin{array}{l}\text { TIPS } \\
(n=78)\end{array}$ & $\begin{array}{l}\text { EPCS } \\
(n=76)\end{array}$ & $\begin{array}{l}\mathrm{P} \\
\text { Value }\end{array}$ \\
\hline $\begin{array}{l}\text { History } \\
\text { Age (yrs): } \\
\text { Mean/median } \\
\text { Range } \\
\mathrm{n} \geq 70 \text { yrs }\end{array}$ & $\begin{array}{l}47.8 / 45 \\
23-75 \\
7\end{array}$ & $\begin{array}{l}49.8 / 47 \\
28-82 \\
9\end{array}$ & $\begin{array}{l}0.21 \\
0.61 \\
0.61\end{array}$ & $\begin{array}{l}49.0 / 47 \\
30-84 \\
1\end{array}$ & $\begin{array}{l}49.1 / 48 \\
31-73 \\
2\end{array}$ & $\begin{array}{l}0.89 \\
0.62 \\
0.35\end{array}$ \\
\hline Male gender, n (\%) & $81(76)$ & $81(78)$ & 0.87 & $56(72)$ & $60(79)$ & 0.65 \\
\hline $\begin{array}{l}\text { Race, } \mathrm{n}(\%) \text { : } \\
\text { Caucasian } \\
\text { Hispanic } \\
\text { Other }\end{array}$ & $\begin{array}{l}53(50) \\
50(47) \\
3(3)\end{array}$ & $\begin{array}{l}58(55) \\
39(37) \\
8(8)\end{array}$ & 0.09 & $\begin{array}{l}33(42) \\
39(50) \\
6(8)\end{array}$ & $\begin{array}{l}37(49) \\
34(45) \\
5(7)\end{array}$ & \\
\hline $\begin{array}{l}\text { Cause of cirrhosis, } \mathrm{n}(\%) \text { : } \\
\text { Alcoholism alone } \\
\text { Hepatitis B or C alone } \\
\text { Alcoholism and hepatitis } \\
\text { Other }\end{array}$ & $\begin{array}{l}58(55) \\
10(9) \\
30(28) \\
8(8)\end{array}$ & $\begin{array}{l}54(51) \\
8(8) \\
33(31) \\
10(9)\end{array}$ & 0.73 & $\begin{array}{l}29(37) \\
4(5) \\
44(56) \\
1(1)\end{array}$ & $\begin{array}{l}24(32) \\
11(14) \\
37(49) \\
4(5)\end{array}$ & 0.083 \\
\hline $\begin{array}{l}\text { Chronic alcoholism, } \mathrm{n}(\%) \text { : } \\
\text { Years of alcoholism, mean (range) } \\
\text { Recent alcohol ingestion } \\
\quad \leq 7 \mathrm{~d}, \mathrm{n}(\%)\end{array}$ & $\begin{array}{l}88(83) \\
22(4-59) \\
55(62)\end{array}$ & $\begin{array}{l}87(83) \\
25(7-54) \\
57(66)\end{array}$ & $\begin{array}{l}1.00 \\
0.56 \\
0.99\end{array}$ & $\begin{array}{l}72(97) \\
21.6(5-45) \\
42(58)\end{array}$ & $\begin{array}{l}61(95) \\
21.6(8-41) \\
34(58)\end{array}$ & $\begin{array}{l}0.66 \\
0.97 \\
0.44\end{array}$ \\
\hline $\begin{array}{l}\text { Past history, } \mathrm{n}(\%) \text { : } \\
\text { Jaundice } \\
\text { Ascites } \\
\text { Portal-systemic encephalopathy } \\
\text { Delirium tremens in alcoholics }\end{array}$ & $\begin{array}{l}61(58) \\
70(66) \\
20(19) \\
23(26)\end{array}$ & $\begin{array}{l}58(55) \\
48(46) \\
30(29) \\
28(31)\end{array}$ & $\begin{array}{l}0.78 \\
0.004^{*} \\
0.11 \\
0.43\end{array}$ & $\begin{array}{l}32(41) \\
40(51) \\
17(22) \\
20(28)\end{array}$ & $\begin{array}{l}42(55) \\
53(70) \\
18(24) \\
21(34)\end{array}$ & $\begin{array}{l}0.11 \\
0.022^{*} \\
0.85 \\
0.45\end{array}$ \\
\hline $\begin{array}{l}\text { Physical examination, } \mathrm{n}(\%) \text { : } \\
\text { Jaundice } \\
\text { Ascites } \\
\text { Portal-systemic encephalopathy } \\
\text { Severe muscle wasting } \\
\text { (2+ or } 3+\text { on } 0-3+\text { scale) } \\
\text { Delirium tremens in alcoholics }\end{array}$ & $\begin{array}{l}45(42) \\
65(61) \\
19(18) \\
50(47) \\
2(2)\end{array}$ & $\begin{array}{l}38(36) \\
54(51) \\
19(18) \\
\\
67(64) \\
2(2)\end{array}$ & $\begin{array}{l}0.40 \\
0.17 \\
1.0 \\
0.02^{*} \\
1.00\end{array}$ & $\begin{array}{l}34(44) \\
37(47) \\
19(24) \\
40(51) \\
1(1)\end{array}$ & $\begin{array}{l}37(49) \\
46(61) \\
17(22) \\
49(64) \\
3(5)\end{array}$ & $\begin{array}{l}0.63 \\
0.11 \\
0.8 \\
0.11 \\
0.33\end{array}$ \\
\hline $\begin{array}{l}\text { PSE Index-median } \\
\text { (interquartile range) }\end{array}$ & $\begin{array}{l}0 \\
(0-0.15)\end{array}$ & $\begin{array}{l}0 \\
(0-0.15)\end{array}$ & 0.46 & $\begin{array}{l}0 \\
(0-0.15)\end{array}$ & $\begin{array}{l}0 \\
(0-0.16)\end{array}$ & 0.44 \\
\hline $\begin{array}{l}\text { Child risk class, } \mathrm{n}(\%) \text { : } \\
\text { A ( } 5 \text { to } 8 \text { points) } \\
\text { B ( } 9 \text { to } 11 \text { points }) \\
\text { C (12 to } 15 \text { points })\end{array}$ & $\begin{array}{l}32(30) \\
46(43) \\
28(26)\end{array}$ & $\begin{array}{l}26(25) \\
49(47) \\
30(29)\end{array}$ & 0.71 & $\begin{array}{l}16(21) \\
39(50) \\
23(29)\end{array}$ & $\begin{array}{l}15(20) \\
37(49) \\
24(32)\end{array}$ & 0.98 \\
\hline Child risk class points, mean/median & $10.1 / 10$ & $10.0 / 10$ & 0.76 & $10.2 / 10$ & $10.6 / 11$ & 0.26 \\
\hline & EST & EPCS & TOTAL & TIPS & EPCS & TOTAL \\
\hline Referred for LT Evaluation, n (\%) & $7(6.6)$ & $6(5.7)$ & $13(6.2)$ & $8(10.5)$ & $2(2.6)$ & $10(6.5)$ \\
\hline $\begin{array}{l}\text { Diagnosis of LT Candidates, } \mathrm{n}(\%) \text { : } \\
\text { Alcoholism } \\
\text { Hepatitis } \\
\text { Alcoholism and hepatitis } \\
\text { Cryptogenic } \\
\text { Autoimmune }\end{array}$ & $\begin{array}{l}3(2.8) \\
2(1.9) \\
0 \\
1(0.9) \\
1(0.9)\end{array}$ & $\begin{array}{l}1(1.0) \\
0 \\
3(2.9) \\
2(1.9) \\
0\end{array}$ & $\begin{array}{l}4(1.9) \\
2(0.9) \\
3(1.4) \\
3(1.4) \\
1(0.5)\end{array}$ & $\begin{array}{l}2(2.6) \\
0 \\
6(7.7) \\
0 \\
0\end{array}$ & $\begin{array}{l}0 \\
0 \\
2(2.6) \\
0 \\
0\end{array}$ & $\begin{array}{l}2(1.3) \\
0 \\
8(5.2) \\
0 \\
0\end{array}$ \\
\hline $\begin{array}{l}\text { Results of LT Evaluation: } \\
\text { LT performed } \\
\text { LT approved but not done } \\
\text { LT denied }\end{array}$ & $\begin{array}{l}4 \\
2 \\
1\end{array}$ & $\begin{array}{l}0 \\
1 \\
5\end{array}$ & $\begin{array}{l}4 \\
3 \\
6\end{array}$ & $\begin{array}{l}4 \\
0 \\
4\end{array}$ & $\begin{array}{l}0 \\
0 \\
2\end{array}$ & $\begin{array}{l}4 \\
0 \\
6\end{array}$ \\
\hline $\begin{array}{l}\text { Reason for denial: } \\
\text { Continued alcoholism } \\
\text { Continued drug abuse } \\
\text { Non-U.S. residence } \\
\text { Continued drug abuse and } \\
\text { non-U.S. residence }\end{array}$ & $\begin{array}{l}0 \\
0 \\
1 \\
0\end{array}$ & $\begin{array}{l}3 \\
1 \\
0 \\
1\end{array}$ & $\begin{array}{l}3 \\
1 \\
1 \\
1\end{array}$ & $\begin{array}{l}2 \\
0 \\
0 \\
2\end{array}$ & $\begin{array}{l}2 \\
0 \\
0 \\
0\end{array}$ & $\begin{array}{l}4 \\
0 \\
0 \\
2\end{array}$ \\
\hline $\begin{array}{l}\text { Years after entry in RCT to final LT } \\
\text { Evaluation-mean (range) }\end{array}$ & $\begin{array}{l}1.0 \\
(0.04-2.35)\end{array}$ & $\begin{array}{l}2.7 \\
(0.02-6.74)\end{array}$ & $\begin{array}{l}1.47 \\
(0.02-6.74)\end{array}$ & $\begin{array}{l}1.5 \\
(0.75-1.5)\end{array}$ & $\begin{array}{l}3.0 \\
(2.0-3.5)\end{array}$ & $\begin{array}{l}2.25 \\
(0.75-3.5)\end{array}$ \\
\hline Approved for LT, n (\%) & $6(5.6)$ & $1(1.0)$ & $7(3.3)$ & $4(5.1)$ & 0 & $4(2.6)$ \\
\hline Years on waiting list-mean (range) & $\begin{array}{l}0.162 \\
(0-0.59)\end{array}$ & $\begin{array}{l}8.96 \\
(n=1)\end{array}$ & & $\begin{array}{l}0.38 \\
(0.5-0.7)\end{array}$ & 0 & \\
\hline Died on waiting list (time) & 0 & 0 & 0 & 0 & 0 & 0 \\
\hline $\begin{array}{l}\text { Survival after LT }(n=) \\
\text { mean (range), yrs }\end{array}$ & $\begin{array}{l}0.204 \\
(0.001-0.805)\end{array}$ & N/A & $\begin{array}{l}0.204 \\
(0.001-0.805)\end{array}$ & $\begin{array}{l}0.4 \\
(0.2-0.6)\end{array}$ & $\mathrm{N} / \mathrm{A}$ & $\begin{array}{l}0.4 \\
(0.2-0.6)\end{array}$ \\
\hline $\begin{array}{l}\text { Survival after evaluation, no LT } \\
\text { mean (range), yrs }(n=)\end{array}$ & $\begin{array}{l}0.237 \\
(0.047-0.586)\end{array}$ & $\begin{array}{l}6.027 \\
(0.017-12.73)\end{array}$ & $\begin{array}{l}4.097 \\
(0.017-12.73)\end{array}$ & $\begin{array}{l}0.6 \\
(0.2-0.8)\end{array}$ & -- & $\begin{array}{l}0.6 \\
(0.2-0.8)\end{array}$ \\
\hline $\begin{array}{l}\text { Survival since entry } \\
\text { mean (range), yrs ( } n=)\end{array}$ & $\begin{array}{l}1.266 \\
(0.088-2.357)\end{array}$ & $\begin{array}{l}8.720 \\
(0.033-13.140)\end{array}$ & $\begin{array}{l}4.706 \\
(0.033-13.140)\end{array}$ & $\begin{array}{l}1.9 \\
(0.75-1.75)\end{array}$ & -- & $\begin{array}{l}1.9 \\
(0.75-1.15)\end{array}$ \\
\hline MELD at LT evaluation-mean (range) & $21.3(7-39)$ & $20.2(7-36)$ & $20.8(7-39)$ & $24(20-30)$ & $26(22-32)$ & $25(20-32)$ \\
\hline
\end{tabular}


Citation: Orloff MJ (2013) Liver Transplantation during Fifty-Three Years' Experience with Randomized Controlled Trials of Emergency Portacaval Shunt for Bleeding Esophageal Varices in Cirrhosis. Surgery Curr Res 3: 140. doi:10.4172/2161-1076.1000140

Page 5 of 13

\begin{tabular}{|l|l|l|l|l|l|}
\hline $\begin{array}{l}\text { MELD at evaluation of LT patients } \\
\text { mean (range) }\end{array}$ & $20.8(17-27)$ & $20.8(17-27)$ & $24(20-28)$ & 24 (20-28) \\
\hline $\begin{array}{l}\text { Child class at LT evaluation: } \\
\text { A }\end{array}$ & 0 & 0 & TOTAL & 0 & 0 \\
B & 1 & 4 & 0 & 0 & 0 \\
C & 6 & 2 & 5 & 0 & 0 \\
\hline
\end{tabular}

*Statistically significant difference

Table 1: Clinical characteristics at time of study entry of patients with cirrhosis and bleeding esophageal varices randomized in RCT No. 1 to Endoscopic Sclerotherapy (EST) or Emergency Portacaval Shunt (EPCS) and in RCT No. 2 to TIPS or Emergency Portacaval Shunt (EPCS) with data on Liver Transplantation (LT).

\section{Results}

\section{Patient characteristics}

The clinical characteristics at the time of enrollment in the two RCTs are summarized in Table 1. The two groups in each RCT were similar in every important characteristic of cirrhosis and BEV. Over $80 \%$ of the patients were entered in the study by transfer from hospitals in the four-county referral area. In over three-fourths of the patients, the cause of cirrhosis was chronic alcoholism, often in combination with hepatitis. The distribution of patients in Child risk classes, determined by Campbell's quantitative modification [22] of the criteria originally proposed by Child and Turcotte [23] was almost identical in the two groups, with 20 to $30 \%$ in risk class A, $43 \%$ and $50 \%$ in risk class B, and $26 \%$ to $29 \%$ in risk class C. The overall Child risk class points in the two groups of patients were essentially identical. Liver biopsies provided histologic proof of cirrhosis in all patients. Upper endoscopy demonstrated sizable esophageal varices in all patients, with active bleeding or evidence of recent acute bleeding in over $90 \%$. Almost twothirds of patients had serologic evidence of hepatitis B or C.

\section{Rapidity of therapy}

Table 3 provides data on rapidity of BEV therapy. The mean and median times from onset of bleeding to entry in the San Diego BEV Study were $<20$ hours in all groups of patients. The mean and median times from onset of bleeding to the start of EST or EPCS or TIPS were $<24$ hours. After initial contact at UCSD Medical Center primary therapy was started in $<8$ hours in most patients and always in less than 24 hours, clearly a reflection of the rapidity with which the diagnostic workup was performed. The mean time from initial contact to primary therapy was always less than 24 hours. Patients who were transferred to UCSD Medical Center from outside facilities on average spent $<12$ hours in the referring hospitals. Before entry into the study, active bleeding had been observed within 4 hours in $84 \%$ of the patients. Without doubt, the study involved evaluation of emergency treatment of acute BEV.

\section{Control of variceal bleeding}

Data on control of variceal bleeding by EEST, TIPS, and EPCS are shown in Table 4. In an effort to obtain temporary control of bleeding while the diagnostic workup was in progress, patients in RCT No. 1 were given a continuous IV infusion of vasopressin, and patients in RCT No. 2 received octreotide intravenously as well as endoscopic sclerotherapy. With regard to immediate and long-term control of variceal bleeding, there was a striking and highly significant difference between EEST and EPCS in RCT No. 1, and between TIPS and EPCS in RCT No. 2 ( $\mathrm{p}<0.001)$. Excluding indeterminate deaths within the first 3 weeks from causes other than bleeding, EEST achieved long-term control of bleeding in only $20 \%$ of patients, and TIPS achieved long-

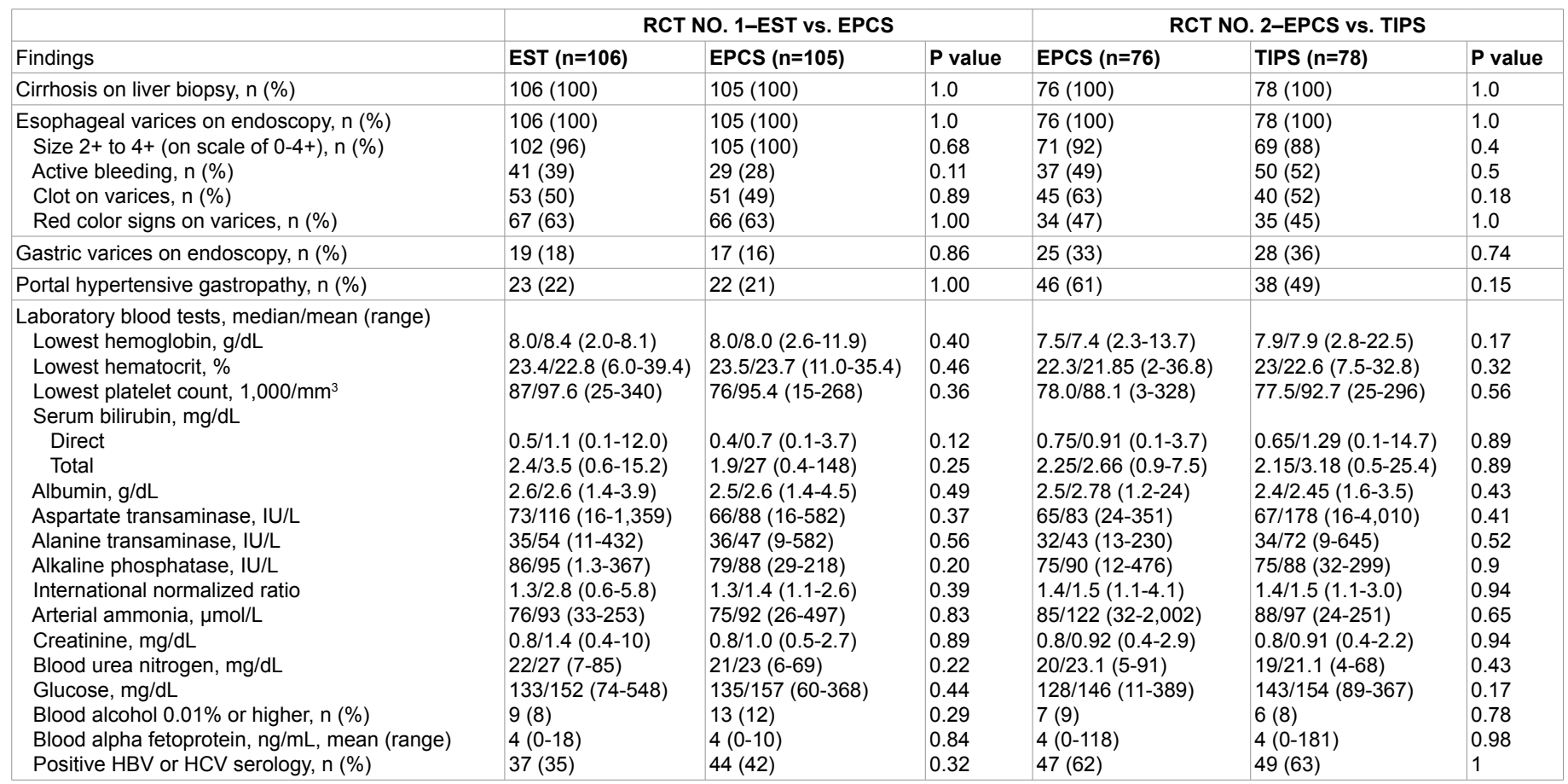

EST-Endoscopic Sclerotherapy; EPCS- Emergency Portacaval Shunt; HBV- Hepatitis B Virus; HCV- Hepatitis C Virus; TIPS-Transjugular Intrahepatic Portosystemic Shunt Table 2: Findings on liver biopsy, endoscopy, and laboratory blood tests at time of study entry in patients with cirrhosis and bleeding esophageal varices randomized in RCT No. 1 to endoscopic sclerotherapy or emergency portacaval shunt and in RCT No. 2 to TIPS or emergency portacaval shunt. 
Citation: Orloff MJ (2013) Liver Transplantation during Fifty-Three Years' Experience with Randomized Controlled Trials of Emergency Portacaval Shunt for Bleeding Esophageal Varices in Cirrhosis. Surgery Curr Res 3: 140. doi:10.4172/2161-1076.1000140

Page 6 of 13

\begin{tabular}{|c|c|c|c|c|c|c|c|c|c|c|}
\hline \multirow[b]{3}{*}{ Hours } & \multicolumn{5}{|c|}{ RCT NO. 1-EST vs. EPCS } & \multicolumn{5}{|c|}{ RCT NO. 2-EPCS vs. TIPS } \\
\hline & \multicolumn{2}{|c|}{ EST $(n=106)$} & \multicolumn{2}{|c|}{$\operatorname{EPCS}(n=105)$} & \multirow[b]{2}{*}{ P Value } & \multicolumn{2}{|c|}{$\operatorname{EPCS}(n=76)$} & \multicolumn{2}{|c|}{ TIPS (n=78) } & \multirow[b]{2}{*}{ P Value } \\
\hline & Median/Mean & Range & Median/Mean & Range & & Median/Mean & Range & Median/Mean & Range & \\
\hline Onset bleeding to study entry & $12 / 19.8$ & $0-144$ & $16 / 19$ & $0-95$ & 0.30 & $20 / 25.4$ & $0-112$ & $17 / 24.2$ & $0.5-84$ & 0.56 \\
\hline Onset bleeding to primary therapy & $15 / 23.3$ & $3-147$ & $19 / 21.5$ & $2.6-100$ & 0.056 & $27.5 / 35.9$ & 8-131.7 & $34.2 / 39.0$ & 11.1-99.4 & 0.08 \\
\hline $\begin{array}{l}\text { Study entry to primary therapy } \\
\mathrm{n}>8 \mathrm{~h} \\
\%>8 \mathrm{~h}\end{array}$ & $\begin{array}{l}2.5 / 3.1 \\
0 \\
0\end{array}$ & $0.8-8$ & $\begin{array}{l}3.4 / 4.4 \\
3 \\
2.9\end{array}$ & $1.4-24.3$ & $<0.001^{*}$ & $\begin{array}{l}9.5 / 10.2 \\
3 \\
3.9\end{array}$ & $2-30.1$ & $\begin{array}{l}14.6 / 14.7 \\
76(97) \\
68(87)\end{array}$ & 2.3-38.4 & $\begin{array}{l}<0.001^{*} \\
1.0 \\
0.5\end{array}$ \\
\hline $\begin{array}{l}\text { Transfer patients, } \mathrm{n} \mathrm{( \% )} \\
\text { Onset of bleeding to entry in referring hospital } \\
\text { Entry into referring hospital to study entry }\end{array}$ & $\begin{array}{l}71(67) \\
4.05 / 10.4 \\
7.2 / 11.8\end{array}$ & $\begin{array}{l}0-127 \\
1.5-53\end{array}$ & $\begin{array}{l}80(76) \\
3.75 / 9.7 \\
8.4 / 11.6\end{array}$ & $\begin{array}{l}0-83.6 \\
0-53\end{array}$ & $\begin{array}{l}0.17 \\
0.92 \\
0.56\end{array}$ & $\begin{array}{l}63(83) \\
6.3 / 11.9 \\
13.0 / 18.4\end{array}$ & $\begin{array}{l}0-120 \\
2.2-110\end{array}$ & $\begin{array}{l}68(87) \\
5.7 / 9.8 \\
10.5 / 16.4\end{array}$ & $\begin{array}{l}0-62 \\
2.6-65.3\end{array}$ & $\begin{array}{l}0.5 \\
0.72 \\
0.42\end{array}$ \\
\hline $\begin{array}{l}\text { Last observation of bleeding to study entry } \\
\quad \leq 4 \mathrm{~h}, \% \text { of group } \\
>4 \mathrm{~h}, \% \text { of group }\end{array}$ & $\begin{array}{l}0 / 1.9 \\
84 \\
16\end{array}$ & $0-32$ & $\begin{array}{l}0 / 2.5 \\
84 \\
16\end{array}$ & $0-30$ & $\begin{array}{l}0.76 \\
0.94 \\
1.00\end{array}$ & $\begin{array}{l}0 / 1.6 \\
67(88) \\
9(12)\end{array}$ & $0-32$ & $\begin{array}{l}0 / 3.6 \\
63(81) \\
15(19)\end{array}$ & $0-84$ & $\begin{array}{l}0.35 \\
0.27\end{array}$ \\
\hline
\end{tabular}

*Statistically significant difference

EPCS-Emergency Portacaval Shunt; EST- Endoscopic Sclerotherapy; TIPS-Transjugular Intrahepatic Portosystemic Shunt

Table 3: Rapidity of therapy for patients with cirrhosis and bleeding esophageal varices in RCT No. 1 (endoscopic sclerotherapy versus emergency portacaval shunt) and RCT No. 2 (TIPS versus emergency portacaval shunt).

\begin{tabular}{|c|c|c|c|c|c|c|}
\hline & RCT NO. 1 & & & RCT NO. 2 & & \\
\hline & EST $(n=106)$ & EPCS $(n=105)$ & P Value & $\operatorname{EPCS}(n=76)$ & TIPS (n=78) & P Value \\
\hline \multicolumn{7}{|l|}{ Control of bleeding, $\mathrm{n}(\%)$} \\
\hline \multicolumn{7}{|l|}{ Temporary during workup } \\
\hline Received vasopressin or octreotide intravenously & $95(90)$ & $80(76)$ & $0.012^{*}$ & $60(83)$ & $71(92)$ & 0.13 \\
\hline Bleeding at start of vasopressin or octreotide infusion & $47(49)$ & $55(69)$ & $0.014^{*}$ & $40(67)$ & $44(71)$ & 0.59 \\
\hline Bleeding decreased or stopped & $36(77)$ & $38(69)$ & 0.51 & $19(48)$ & $24(58)$ & 0.66 \\
\hline \multicolumn{7}{|c|}{ Permanent by EST or EPCS (RCT No. 1) or by TIPS (RCT No. 2) } \\
\hline Indeterminate, nonbleeding death $\leq 14 \mathrm{~d}$ & $4(4)$ & $12(11)$ & $0.040^{*}$ & $16(21)$ & $8(10)$ & 0.078 \\
\hline $\begin{array}{l}14 \mathrm{~d} \\
\text { Successul control excluaing indeterminates for at leas }\end{array}$ & $22(21)$ & $93(100)$ & $<0.001^{*}$ & $60(100)$ & $58(83)$ & $<0.001^{*}$ \\
\hline $30 \mathrm{~d}$ & $20(20)$ & $89(100)$ & $<0.001^{*}$ & $57(95)$ & $56(80)$ & $0.017^{*}$ \\
\hline$>30 \mathrm{~d}$ & $20(20)$ & $89(100)$ & $<0.001^{*}$ & 57 (95) & $54(77)$ & $0.005^{*}$ \\
\hline Declaration of primary therapy failure, $\mathrm{n}(\%)$ & $81(79)$ & $0(0)$ & $<0.001^{*}$ & & & \\
\hline Required $\geq 6 \cup$ PRBC in first $7 \mathrm{~d}$ & $15(19)$ & - & & $0(0)$ & $9(13)$ & $0.003^{*}$ \\
\hline Required $\geq 8 \cup \mathrm{PRBC}$ in any $12 \mathrm{mo}$ & $47(58)$ & - & & $2(3)$ & $33(47)$ & $<0.001^{*}$ \\
\hline \multicolumn{7}{|l|}{ Recurrent variceal bleeding after variceal } \\
\hline obliteration was declared & $27(33)$ & - & & $0(0)$ & $32(46)$ & $<0.001^{*}$ \\
\hline More than one criterion for failure & $8(10)$ & - & & $0(0)$ & $19(27)$ & $<0.001^{*}$ \\
\hline \multicolumn{7}{|l|}{ PRBC transfusion, $U$, mean/median (range) } \\
\hline Index hospitalization & & & & & & \\
\hline Before primary therapy & $4.5 / 4(2-12)$ & $5.8 / 5(2-17)$ & $<0.001^{*}$ & $5 / 5.6(0-14)$ & $5 / 6.3(0-30)$ & 0.83 \\
\hline During primary therapy & $0.6 / 0(0-7)$ & $6.3 / 3(0-68)$ & $<0.001^{*}$ & $3.5 / 5.4(0-32)$ & $0 / 0.4(0-6)$ & $<0.001^{*}$ \\
\hline "Catch-up" after primary therapy & $0.2 / 0(0-4)$ & $1.2 / 0(0-21)$ & $0.001^{*}$ & $0 / 0.8(0-15)$ & $0 / 0.2(0-4)$ & 0.056 \\
\hline \multicolumn{7}{|l|}{ Posttherapy bleeding } \\
\hline Variceal & $4.4 / 0(0-37)$ & $0 / 0(0-0)$ & $<0.001^{*}$ & $0 / 1.1(0-46)$ & $0 / 2.6(0-29)$ & $<0.001^{*}$ \\
\hline Nonvariceal & $0.3 / 0(0-11)$ & $1.8 / 0(0-29)$ & 0.059 & $0 / 1.1(0-21)$ & $0 / 0.1(0-6)$ & $0.020^{*}$ \\
\hline Total PRBC units & $10.0 / 7(2-44)$ & $15.0 / 10(2-81)$ & $<0.001^{*}$ & $9 / 14.0(0-85)$ & $7 / 9.8(2-42)$ & $0.010^{*}$ \\
\hline \multicolumn{7}{|l|}{ Readmissions for bleeding } \\
\hline Variceal bleeding & $6.8 / 2(0-60)$ & $0.4 / 0(0-26)$ & $<0.001^{*}$ & $0 / 0.2(0-14)$ & $6 / 6.6(0-36)$ & $<0.001^{*}$ \\
\hline Nonvariceal bleeding & $3.8 / 0(0-38)$ & $3.5 / 0(0-33)$ & 0.23 & $0 / 0.4(0-10)$ & $0 / 0.5(0-11)$ & 0.62 \\
\hline Total PRBC units & $10.6 / 7(0-60)$ & $3.9 / 0(0-33)$ & $<0.001^{*}$ & $0 / 06.9(0-14)$ & $6 / 7.0(0-36)$ & $<0.001^{*}$ \\
\hline Total PRBC units for variceal bleeding & $15.8 / 14(2-64)$ & $13.6 / 10(2-73)$ & $0.037^{*}$ & $8 / 11.7(0-85)$ & $14 / 15(3-47)$ & $<0.001^{*}$ \\
\hline Overall survival $(\mathrm{Pr} / 95 \% \mathrm{Cl})$ & & & $<0.001^{*}$ & & & $<0.001^{*}$ \\
\hline $30-d$ & $0.88(0.81,0.94)$ & $0.87(0.80,0.93)$ & & $0.77(0.68,0.87)$ & $0.78(0.70,0.88)$ & 0.96 \\
\hline $1-y r$ & $0.72(0.64,0.81)$ & $0.81(0.74,0.89)$ & & $0.75(0.65,0.85)$ & $0.55(0.45,0.67)$ & $0.022^{*}$ \\
\hline $2-y r$ & $0.54(0.45,0.64)$ & $0.77(0.70,0.86)$ & & $0.68(0.58,0.79)$ & $0.49(0.39,0.61)$ & $0.022^{*}$ \\
\hline $3-y r$ & $0.44(0.36,0.55)$ & $0.75(0.67,0.84)$ & & $0.65(0.55,0.77)$ & $0.36(0.27,0.48)$ & $0.001^{*}$ \\
\hline $4-y r$ & $0.35(0.27,0.45)$ & $0.74(0.66,0.83)$ & & $0.63(0.53,0.75)$ & $0.23(0.15,0.35)$ & $<0.001^{*}$ \\
\hline $5-y r$ & $0.21(0.14,0.30)$ & $0.73(0.65,0.82)$ & & $0.61(0.51,0.73)$ & $0.20(0.13,0.31)$ & $<0.001^{*}$ \\
\hline $7-\mathrm{yr}$ & & & & $0.52(0.41,0.66)$ & $0.15(0.09,0.28)$ & $<0.001^{*}$ \\
\hline $10-y r$ & $0.09(0.05,0.17)$ & $0.46(0.37,0.56)$ & & -- & -- & \\
\hline $15-y r$ & $0.09(0.05,0.17)$ & $0.46(0.37,0.56)$ & & - & - & \\
\hline Median survival, yr (95\% Cl) & $2.48(1.58,3.76)$ & $6.17(5.60,10.37)$ & & $>10(5.1>10)$ & $1.99(0.52,2.8)$ & \\
\hline \multicolumn{7}{|l|}{ Survival by Child risk class $(\mathrm{Pr} / 95 \% \mathrm{Cl})$} \\
\hline \multicolumn{7}{|l|}{ 5-yr Child class (PR/95\% $\mathrm{Cl}$ ) } \\
\hline$A$ & $0.38(0.24,0.59)$ & $0.89(0.83,1.00)$ & $0.003^{*}$ & $0.85(0.68,1)$ & $0.38(0.20,0.71)$ & $0.006^{*}$ \\
\hline B & $0.15(0.08,0.30)$ & $0.76(0.64,0.89)$ & $<0.001^{*}$ & $0.65(0.51,0.82)$ & $0.22(0.12,0.41)$ & $<0.002^{*}$ \\
\hline $\mathrm{C}$ & $0.11(0.04,0.31)$ & $0.57(0.41,0.78)$ & $0.005^{*}$ & $0.42(0.26,0.67)$ & $0.04(0.01,0.30)$ & $0.016^{*}$ \\
\hline \multicolumn{7}{|l|}{10 -yrs Child class (PR/95\% Cl) } \\
\hline A & $0.18,(0.08,0.38)$ & $0.62(0.45,0.83)$ & $0.003^{*}$ & $0.76(0.55,1)$ & $0.30(0.20,0.71)$ & $0.008^{*}$ \\
\hline $\mathrm{B}$ & $0.07(0.02,0.20)$ & $0.45(0.33,0.61)$ & $<0.001^{*}$ & $0.51(0.36,0.71)$ & $0.15(0.06,0.41)$ & $0.003^{*}$ \\
\hline $\mathrm{C}$ & $0.04(0.01,0.25)$ & $0.33(0.20,0.55)$ & $0.005^{*}$ & $0.42(0.26,0.67)$ & $0.04(0.01,0.30)$ & $0.016^{*}$ \\
\hline \multicolumn{7}{|l|}{ Median survival, yr $(95 \% \mathrm{Cl})$} \\
\hline$A$ & $4.62(4.08,6.34)$ & $10.43(>5.92)$ & $0.003^{*}$ & $>9.37(>9.37>9.37)$ & $3.46(0.51,8.05)$ & $0.008^{*}$ \\
\hline$B$ & $2.61(1.65,3.96)$ & $6.19(>5.44)$ & $<0.001^{*}$ & $>9.80(5.09>9.80)$ & $2.12(1.03,3.00)$ & $<0.001^{*}$ \\
\hline $\mathrm{C}$ & $0.58(0.12,2.36)$ & $5.30(0.70,10.16)$ & $0.005^{*}$ & $1.25(0.06>9.66)$ & $0.08(0.04,1.99$ & $0.016^{*}$ \\
\hline
\end{tabular}


Citation: Orloff MJ (2013) Liver Transplantation during Fifty-Three Years' Experience with Randomized Controlled Trials of Emergency Portacaval Shunt for Bleeding Esophageal Varices in Cirrhosis. Surgery Curr Res 3: 140. doi:10.4172/2161-1076.1000140

\begin{tabular}{|c|c|c|c|c|c|c|}
\hline \multicolumn{7}{|l|}{$\begin{array}{l}\text { Survival by direct UCSD admission versus transfer from } \\
\text { outside UCSD }(\operatorname{Pr} / 95 \% \mathrm{Cl})\end{array}$} \\
\hline Direct UCSD admission & $0.38(0.24,0.59)$ & $0.89(0.83,1.00)$ & $0.003^{*}$ & $0.85(0.68,1)$ & $0.38(0.20,0.71)$ & $0.006^{*}$ \\
\hline $5 \mathrm{yr}$ & $0.15(0.08,0.30)$ & $0.76(0.64,0.89)$ & $<0.001^{*}$ & $0.65(0.51,0.82)$ & $0.22(0.12,0.41)$ & $<0.002^{*}$ \\
\hline $7 \mathrm{yr}$ & $0.11(0.04,0.31)$ & $0.57(0.41,0.78)$ & $0.005^{*}$ & $0.42(0.26,0.67)$ & $0.04(0.01,0.30)$ & $0.016^{\star}$ \\
\hline $10 \mathrm{yr}$ & & & & & & \\
\hline Transfer from outside UCSD & $0.18,(0.08,0.38)$ & $0.62(0.45,0.83)$ & $0.003^{*}$ & $0.76(0.55,1)$ & $0.30(0.20,0.71)$ & $0.008^{*}$ \\
\hline $5 \mathrm{yr}$ & $0.07(0.02,0.20)$ & $0.45(0.33,0.61)$ & $<0.001^{*}$ & $0.51(0.36,0.71)$ & $0.15(0.06,0.41)$ & $0.003^{*}$ \\
\hline $\begin{array}{l}7 \mathrm{yr} \\
10 \mathrm{yr}\end{array}$ & $0.04(0.01,0.25)$ & $0.33(0.20,0.55)$ & $0.005^{*}$ & $0.42(0.26,0.67)$ & $0.04(0.01,0.30)$ & $0.016^{*}$ \\
\hline Mean survival, yr $(95 \% \mathrm{Cl})$ & $4.62(4.08,6.34)$ & $10.43(>5.92)$ & $0.003^{*}$ & $>9.37(>9.37>9.37)$ & $3.46(0.51,8.05)$ & $0.008^{*}$ \\
\hline Direct UCSD admission & $2.61(1.65,3.96)$ & $6.19(>5.44)$ & $<0.001^{*}$ & $>9.80(5.09>9.80)$ & $2.12(1.03,3.00)$ & $<0.001^{*}$ \\
\hline Transfer from outside UCSD & $0.58(0.12,2.36)$ & $5.30(0.70,10.16)$ & $0.005^{\star}$ & $1.25(0.06>9.66)$ & $0.08(0.04,1.99)$ & $0.016^{*}$ \\
\hline $\begin{array}{l}\text { Survival by direct UCSD admission versus transfer from } \\
\text { outside UCSD (Pr/95\% Cl) } \\
\text { Direct UCSD admission } \\
5 \mathrm{yr} \\
7 \mathrm{yr} \\
10 \mathrm{yr} \\
\text { Transfer from outside UCSD } \\
5 \mathrm{yr} \\
7 \mathrm{yr} \\
10 \mathrm{yr} \\
\text { Mean survival, yr }(95 \% \mathrm{Cl}) \\
\text { Direct UCSD admission } \\
\text { Transfer from outside UCSD }\end{array}$ & $\begin{array}{l}0.17(0.08,0.36) \\
-- \\
0.11(0.05,0.29)\end{array}$ & $\begin{array}{l}0.58(0.42,0.80) \\
-- \\
0.52(0.27,0.66) \\
0.79(0.70,0.88) \\
-- \\
0.45(0.36,0.58) \\
\\
5.28(>2.80) \\
6.25(5.81,11.03)\end{array}$ & 0.48 & $\begin{array}{l}0.46(0.26,0.83) \\
0.31(0.14,0.70) \\
-- \\
0.64(0.53,0.77) \\
0.57(0.46,0.72)\end{array}$ & $\begin{array}{l}0.20(0.06,0.69) \\
0.10(0.02,0.64) \\
-- \\
0.20(0.12,0.32) \\
0.17(0.10,0.30)\end{array}$ & 0.13 \\
\hline $\begin{array}{l}\text { Rescue portacaval shunt in EST group-effect on median survival, yr } \\
(95 \% \text { Cl) and rescue portacaval shunt in TIPS group } \\
\text { Successful EST ( } n=21) \text { or TIPS }(n=15) \\
\text { Failed EST }(n=81) \text { or failed TIPS }(n=55) \\
\text { Rescue shunt-failed EST }(n=50) \text { or rescue shunt in failed TIPS }(n=8) \\
\text { No rescue shunt-failed EST }(n=31) \text { or failed TIPS }(n=39) \\
\text { Overall survival: rescue shunt }(n=50) \text { versus primary } \\
\text { EPCS ( } n=105) \text { in RCT No. } 1 \text { and rescue shunt }(n=8) \text { versus } \\
\text { Primary EPCS }(n=64) \text { in RCT No. } 2 \\
\text { Postoperative survival: rescue shunt }(n=50) \text { versus } \\
\text { primary EPCS }(n=105) \\
\text { 10-yr Child class (Pr/95\% Cl) } \\
\text { A } \\
\text { B } \\
\text { C } \\
\text { Median survival, yr }(95 \% \text { Cl) } \\
\text { A } \\
\text { B } \\
\text { C }\end{array}$ & $\begin{array}{l}2.76(1.52,7.35) \\
2.71(1.65,3.92) \\
3.01(1.65,4.34) \\
2.36(0.72,4.34) \\
\\
3.01(1.65,4.34) \\
1.99(1.34,3.73) \\
\\
0.18(0.08,0.38) \\
0.07(0.02,0.20) \\
0.04(0.01,0.25)\end{array}$ & $\begin{array}{l}-- \\
-- \\
-- \\
6.18(5.61,10.38) \\
6.18(5.61,10.38) \\
0.62(0.45,0.83) \\
0.45(0.33,0.61) \\
0.33(0.20,0.55)\end{array}$ & $\begin{array}{l}0.48 \\
0.098 \\
<0.001^{*} \\
<0.001^{*} \\
0.003^{*} \\
<0.001^{*} \\
0.005^{*}\end{array}$ & $\begin{array}{l}-- \\
-- \\
-- \\
>10(5.1>10) \\
>10(5.1>10)\end{array}$ & $\begin{array}{l}3.75(1.15>7.18) \\
2.04(0.92,2.88) \\
>8.06(3.93>8.06) \\
1.03(0.50,2.67) \\
>8.06(3.93>8.06) \\
>8.04(3.08>8.04)\end{array}$ & $\begin{array}{l}0.95 \\
0.98 \\
0.98 \\
0.67\end{array}$ \\
\hline $\begin{array}{l}\text { Survival by direct UCSD admission versus transfer from } \\
\text { outside UCSD (Pr/95\% Cl) } \\
\text { Direct UCSD admission } \\
5 \mathrm{yr} \\
10 \mathrm{yr} \\
\text { Transfer from outside UCSD } \\
5 \mathrm{yr} \\
10 \mathrm{yr} \\
\text { Mean survival, yr }(95 \% \mathrm{Cl}) \\
\text { Direct UCSD admission } \\
\text { Transfer from outside UCSD }\end{array}$ & $\begin{array}{l}0.17,(0.08,0.36) \\
0.11(0.05,0.29) \\
0.24(0.16,0.36) \\
0.08(0.03,0.18) \\
\\
2.71(0.72,4.51) \\
2.45(1.59,3.76)\end{array}$ & $\begin{array}{l}0.58(0.42,0.80) \\
0.52(0.27,0.66) \\
\\
0.79(0.70,0.88) \\
0.45(0.36,0.58) \\
\\
5.28(>2.80) \\
6.25(5.81,11.03)\end{array}$ & 0.48 & $\begin{array}{l}0.46(0.26,0.83) \\
\text { 7-yr: } 0.31(0.14,0.70) \\
0.64(0.53,0.77) \\
7-y r: 0.57(0.46,0.72) \\
\\
1.25(0.04>9.79) \\
>10(5.18>10)\end{array}$ & $\begin{array}{l}0.20(0.06,0.69) \\
0.10(0.02,0.64) \\
0.20(0.12,0.32) \\
0.17(0.10,0.30) \\
\\
1.03(0.49,>7.13) \\
1.99(0.51,3.0)\end{array}$ & 0.13 \\
\hline $\begin{array}{l}\text { Rescue portacaval shunt in EST group (RCT No. 1) } \\
\text { Or TIPS (RCT No. 2)-effect on median survival, yr }(95 \% \text { CI) } \\
\text { Successful EST }(n=21) \text { or TIPS }(n=15) \\
\text { Failed EST ( } n=81) \text { or TIPS }(n=55) \\
\text { Rescue shunt-failed EST }(n=50) \text { or failed TIPS }(n=8) \\
\text { No rescue shunt-failed EST }(n=31) \text { or failed TIPS }(n=39) \\
\text { Overall survival: rescue shunt }(n=50) \text { versus primary } \\
\text { EPCS ( } n=105) \text { in RCT No. } 1 \text {, rescue shunt }(n=8) \\
\text { versus primary EPCS in RCT No. } 2 \\
\text { Postoperative survival: rescue shunt }(n=50) \text { versus } \\
\text { primary EPCS }(n=105) \text { in RCT No. } 1 \text {, rescue shunt }(n=8) \\
\text { versus primary EPCS }(n=76) \text { in RCT No. } 2\end{array}$ & $\begin{array}{l}2.76(1.52,7.35) \\
2.71(1.65,3.92) \\
3.01(1.65,4.34) \\
2.36(0.72,4.34) \\
3.01(1.65,4.34) \\
1.99(1.34,3.73)\end{array}$ & $\begin{array}{l}-- \\
-- \\
-- \\
-- \\
6.18(5.61,10.38) \\
6.18(5.61,10.38)\end{array}$ & $\begin{array}{l}<0.001^{*} \\
<0.001^{*}\end{array}$ & $\begin{array}{l}-- \\
-- \\
-- \\
-- \\
>10(5.1>10) \\
-- \\
>10(5.1>10)\end{array}$ & $\begin{array}{l}3.75(1.15,>7.18) \\
2.04(0.92,2.88) \\
>8.06(3.98,>8.06) \\
>8.06(3.93,>8.06) \\
1.03(0.50,2.67) \\
>8.06(3.98,>8.06 \\
\\
>8.04(3.08,>8.04)\end{array}$ & $\begin{array}{l}0.95 \\
0.001^{*}\end{array}$ \\
\hline
\end{tabular}

Pr-probability; PRBC, Packed Red Blood Cells; UCSD-University of California-San Diego.

*Statistically significant difference

Table 4: Control of bleeding and survival in RCT No. 1 (endoscopic sclerotherapy versus emergency portacaval shunt) and in RCT No. 2 (emergency portacaval shunt versus TIPS) in patients with bleeding esophageal varices. 
term control of bleeding in only $22 \%$. In contrast, EPCS promptly and permanently controlled bleeding in $100 \%$ of patients in RCT No. 1, and in $97 \%$ of patients in RCT No. 2. Failure of EEST in RCT No. 1 and of TIPS in RCT No. 2 was declared according to the criteria established by the study protocol.

The requirement for PRBC transfusions reflects the success or failure of treatment of BEV and is summarized in Table 4. In each RCT, patients in the EPCS group required almost no blood transfusions for recurrent BEV. In contrast, patients treated by EEST and those treated by TIPS had repeated readmissions to the hospital for recurrent BEV that required blood transfusions.

\section{Survival}

Table 4 summarizes data on survival and Figure 2 shows long-term Kaplan-Meier estimated survival plots for EEST and EPCS in RCT No. 1 , and Figure 3 shows estimated survival for TIPS and EPCS in RCT No. 2. The 30 -day survival rates of $88 \%$ and $87 \%$, respectively, for EEST and EPCS in RCT No. 1, and 77\% and 78\% for EPCS and TIPS in RCT No. 2 were essentially identical. Subsequently, however, there were highly significant differences of the two study groups at all time intervals in each RCT. In RCT No. 1, the 1-, 5-, 10-, and 15-years survival rates in the EEST group were $72 \%, 21 \%, 9 \%$, and $9 \%$, respectively, and in the EPCS group were $81 \%, 73 \%, 46 \%$, and $46 \%$, respectively $(\mathrm{p}<0.001)$. In RCT No. 2, the 1-, 3-, 5-, and 7-year survival rates in the TIPS group were $55 \%, 36 \%, 20 \%$, and $15 \%$, respectively, and in the EPCS group were $75 \%, 65 \%, 61 \%$, and $52 \%$, respectively $(\mathrm{p}<0.001)$. Median survival following TIPS was 1.9 years while median survival following EPCS was greater than 10 years. As anticipated, survival rates were related to effectiveness in control of bleeding as well as to the severity of liver disease as expressed by Child risk class upon entry in each RCT, and shown in Table 4 and Figure 2.

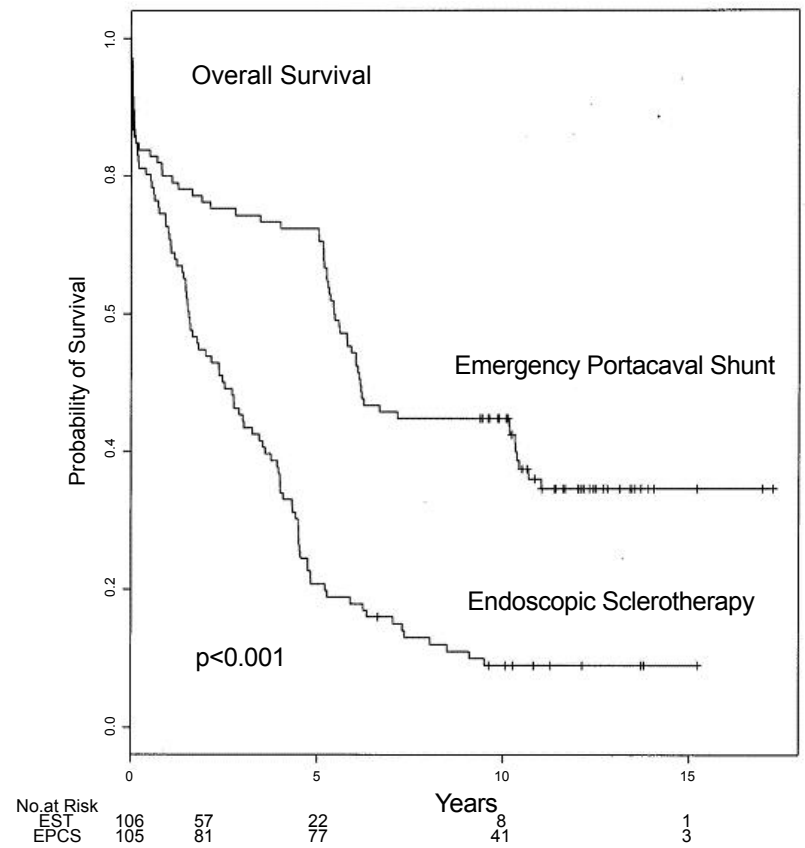

Figure 2: Kaplan-Meier estimates of overall survival after endoscopic sclerotherapy (EST) $(n=106)$ and Emergency Portacaval Shunt (EPCS) $(n=105)$.

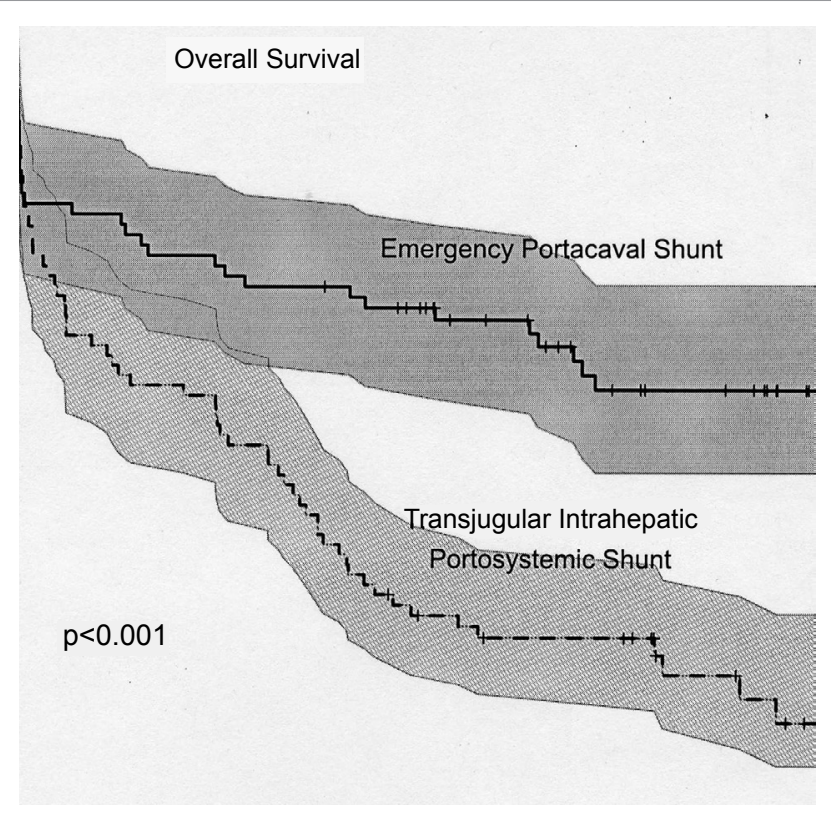

Figure 3: Kaplan-Meier estimates of overall survival following EPCS $(n=76$ ) and TIPS $(n=78)$. Confidence intervals are represented by shaded areas.

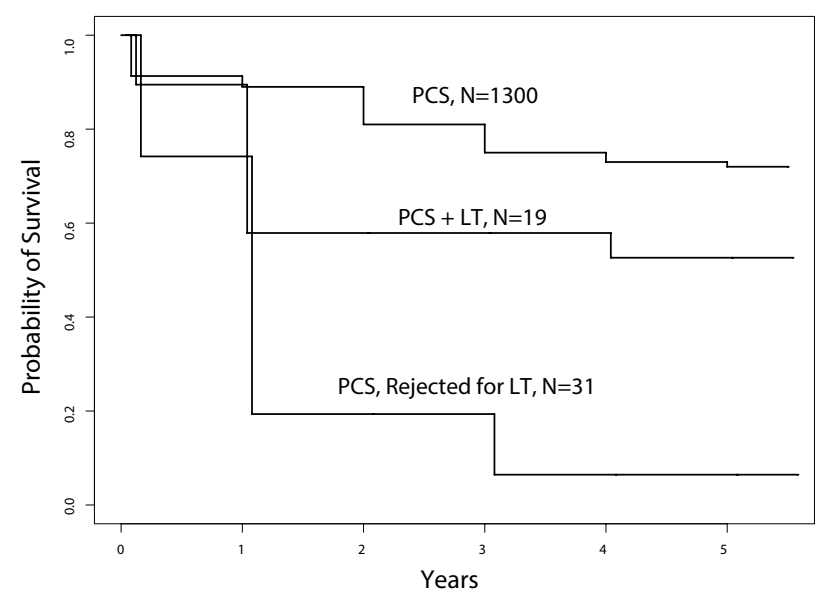

Figure 4: Kaplan-Meier estimates of survival of 1300 unrandomized PCS patients including survival of patients who underwent LT and patients who were rejected for $\mathrm{LT}$.

In striking contrast, the 1- and 5-years LT survival rates were $0.68 \%$ and 0 , respectively, in RCT No. 1 , and $0.4 \%$ and 0 in RCT No. 2.

\section{Operative EPCS data}

Direct side-to-side PCS was performed in $94 \%$ of the 105 patients randomized to EPCS in RCT No. 1, and in $93 \%$ of the 76 patients randomized to EPCS in RCT No. 2. The remaining 6-7\% of patients received a direct end-to-side PCS. Mean operative time for EPCS in RCT No. 1 was 4.0 hours and in RCT No. 2 was 3.8 hours. Estimated blood loss during EPCS in these patients with active variceal bleeding, averaged 3,094 $\mathrm{ml}$ in RCT No. 1 and 1,600 $\mathrm{ml}$ in RCT No. 2. Accordingly, the operative requirement for PRBC transfusion in these actively bleeding patients was 6.3 units in RCT No. 1 and 3.0 units in RCT No. 2. The PV-IVC pressure gradient before the shunt averaged $244 \mathrm{~mm}$ saline in RCT No. 1 and $241 \mathrm{~mm}$ saline in RCT No. 2. EPCS reduced the PV-IVC gradient to a mean $17 \mathrm{~mm}$ in RCT No. 1 and 20 
$\mathrm{mm}$ in RCT No. 2. EPCS promptly eliminated portal hypertension in all patients and stopped the bleeding. Importantly, the PCS remained permanently patent throughout life in $98 \%$ of the patients in RCT No. 1 and $97 \%$ of the patients in RCT No. 2 .

\section{Operative TIPS data}

Median TIPS operative time was $3 \mathrm{hrs}$ (range 1.25 to $25 \mathrm{hrs).} \mathrm{The}$ median PV-IVC pressure gradient before TIPS was $22 \mathrm{mmHg}$ (range 10 to $42 \mathrm{mmHg}$ ), and after TIPS it was reduced to $7 \mathrm{mmHg}$ (range 0 to $17 \mathrm{mmHg}$ ). The mean number of Wallstents placed in the TIPS was 1.24 (range 0 to 3), with a diameter of $12 \mathrm{~mm}$ in $96 \%$ of patients and $10 \mathrm{~mm}$ in $4 \%$ and a length of $9 \mathrm{~cm}$ in $69 \%$ of patients and $6 \mathrm{~cm}$ in the remainder. Balloon diameter was 10 or $12 \mathrm{~mm}$ in $96 \%$ of subjects. The median number of needle passes required to enter the PV was 5 (range 1 to 40). During the TIPS procedure, the mean requirement for PRBC transfusions was 0.53 units (range 0 to 6 ). At the conclusion of the procedure, TIPS was considered a technical success in $87 \%$ of the patients, a failure in $11 \%$, and indeterminate in $2.7 \%$.

\section{TIPS technical success or failure during follow-up}

TIPS-specific follow-up by color Doppler sonography, angiography, EGD, and regular clinical examinations was conducted rigorously. Since almost one-fourth of the patients died during the first 3 weeks postoperatively, the durability of TIPS was analyzed in the patients who survived for 21 days or more. TIPS stenosis or occlusion was demonstrated in 38 (84\%) of these patients. They developed a mean 2.1 episodes of TIPS stenosis or occlusion (standard deviation 1.80, range $0-9)$. TIPS stenosis or occlusion was demonstrated 46 times in the first post-entry year, 26 times in the second year after entry, and 26 times in patients who survived for three or more years. Twenty-four of these 38 patients with TIPS malfunction, or $63 \%$, underwent revision of the TIPS by balloon angioplasty or insertion of one or more additional stents. The revisions failed in $80 \%$ of the patients, and success was indeterminate in an additional $8 \%$. The durability of TIPS was disappointing.

\section{Portal-systemic encephalopathy (PSE)}

The important complication of PSE was analyzed and reported in detail in our recent publications, which should be consulted by the reader $[24,25]$. Recurrent PSE was defined as two or more episodes of PSE after primary therapy in patients who survived 30 days and left the hospital. In RCT No. 1, recurrent PSE developed in 35\% of the EEST group and 15\% of the EPCS group, a highly significant difference $(\mathrm{p}=0.001)$. In RCT No. 2, recurrent PSE developed in $61 \%$ of the patients treated by TIPS, compared to $21 \%$ of the patients who underwent EPCS. The three-fold greater incidence of PSE in the TIPS group was highly significant $(\mathrm{p}<0.001)$.

\section{Direct cost of care}

The total post-index charges were significantly greater in patients who were treated by EEST or TIPS compared to those who underwent EPCS $(\mathrm{p}<0.001)$, and the total overall charges for emergency and longterm care required over a number of years were greater in patients who received emergency followed by long-term repetitive EEST, or by TIPS related to length of survival and, therefore, days or years during which care was required. EPCS was significantly less expensive than EEST or TIPS in every aspect of care except for the index admission. Charges for post-index care per year in the EEST and EPCS groups, respectively, were a mean $\$ 108,500$ versus $\$ 25,100(p<0.001)$. Total overall charges for care of patients who entered RCT No. 1 were a mean $\$ 168,100$ per year in the EEST group, versus $\$ 39,400$ per year in the EPCS group $(\mathrm{p}<0.001)$.
Of particular note were the charges in patients who failed EEST and underwent a rescue PCS. Charges for such patients were significantly higher than the charges required by EEST patients who did not have a rescue shunt as well as by the patients who underwent EPCS. This finding is noteworthy because the main use of surgical shunts in recent years in the USA and abroad has been as elective rescue treatment for failure of endoscopic therapy and other forms of treatment of esophageal varices. Our study indicates that such use of surgical shunts is not only substantially less effective than EPCS, but also is much more costly.

The reasons why EPCS was less costly than EEST and TIPS are very likely a consequence of differences in effectiveness of emergency treatment of BEV. The most important determinants of effectiveness of therapy are survival rate, control of bleeding, and incidence of recurrent PSE. As we have observed in our recent reports, compared to EEST and TIPS, EPCS produced a significantly greater survival rate, was much more effective in controlling bleeding, and was followed by less than one half the incidence of PSE.

\section{Liver transplantation in RCT No. 1 and RCT No. 2}

Of the 211 patients in RCT No. 1, only 13 (6\%) were ultimately referred for LT, mainly because of progressive liver failure; only 7 (3\%) were approved for LT and only 4 (2\%) underwent LT. The 1- and 5-years LT survival rates were $0.68 \%$ and 0 , respectively, compared with $81 \%$ and $73 \%$ survival rates, respectively, after EPCS. Of the 154 patients in RCT No. 2, only 10 (6.5\%) were ultimately referred for LT, mainly because of progressive liver failure. Eight of the 10 patients were in the TIPS group. Only 4 (2.6\%) were approved for LT and only these 4 (2.6\%) underwent LT. Thus, only $2 \%$ of the total 365 randomized patients with BEV underwent LT. The 1- and 5-years LT survival rates in RCT No. 2 were $0.4 \%$ and 0 , respectively, compared with $75 \%$ and $61 \%$ survival rates, respectively, after EPCS.

We concluded that if bleeding is permanently controlled, as occurred invariably following EPCS, cirrhotic patients with BEV seldom require LT. Should LT be required in patients with PCS, although technically more demanding, numerous studies have shown that PCS does not increase mortality or complications. Our experience confirmed that conclusion.

Table 1 summarizes data on the 23 patients who were ultimately referred for LT from among the 365 patients (6\%) randomized in RCT No. 1 and RCT No. 2. Figure 2 shows Kaplan-Meier survival plots of the patients who underwent LT and the patients who were evaluated for but did not undergo LT, and compares their survival curves with the Kaplan-Meier curves showing the overall survival of patients who received EST or TIPS or EPCS in the RCTs. As was true of the overall survival of patients in the EPCS group, patients from this group who were evaluated for LT lived more than 8 years, even though they did not undergo LT. In contrast, as was true of the EST and TIPS groups in general, patients from these groups who were evaluated for or underwent LT had a mean survival of 1.5 years or less.

\section{Liver transplantation in the unrandomized portacaval shunt patients}

All 1300 unrandomized patients had cirrhosis proven by biopsy and bleeding varices proven by endoscopy. The cause of cirrhosis in $85 \%$ of the patients was chronic alcoholism without or with serologically proven chronic viral hepatitis. Quantitative Child's risk classes were A, $11 \%$; B, 50\%; and C, 39\%. Serious risk factors on admission or past history were ascites in $64 \%$, jaundice in $58 \%$, PSE in $36 \%$, and severe muscle wasting in $49 \% .90 \%$ of the patients had end-stage cirrhosis. 
Indications for LT after PCS were declining liver function in the presence of end-stage cirrhosis. Only patients who had been abstinent for 6 or more months were accepted as candidates for LT.

Only 50 of the 1300 patients $(3.8 \%)$ were referred for LT. 19 of the 1300 (1.5\%), all in Child's class B (26\%) or C (74\%), underwent LT. 12 were EPCS patients and 7 were elective PCS patients. The remaining 31 patients were rejected for LT because of resumption of alcoholism, substance abuse, infection, and various other serious comorbidity.

The 30days, 5-years, and 10-years survival rates, respectively, were $83 \%, 67 \%$, and $67 \%$ after emergency PCS, and $98 \%, 76 \%$, and $71 \%$ after elective PCS. Following LT, the 30-days and 5-years survival rates were $89 \%$ and $53 \%$, respectively. The 5 -years survival rate of the 31 patients who were proposed but rejected for LT was only $7 \%$. PCS did not increase the mortality rate or complications of subsequent LT compared with the reports in the literature of that time period and with a large group of unshunted cirrhotic patients who underwent LT. Figure 4 compares 5-years Kaplan-Meier survival plots of the 1300 PCS patients with both the PCS patients who underwent LT and the PCS patients who were rejected for LT.

Regular follow-up was achieved in $100 \%$ of the patients for 5 years and $97 \%$ for 10 years. During those time periods, all but 2 of the 1300 patients remained free of recurrent BEV and the PCS remained permanently patent in all but 2 patients (99.8\%). Recurrent PSE was observed in $10 \%$ of the patients. Liver function improved in $76 \%$ of the patients and, after 5 years, only $6 \%$ of the patients were in Child's class C, compared to $39 \%$ on admission.

\section{Discussion}

The two RCTs of emergency treatment of BEV that are the substance of this report were unique in a number of important respects. They involved a large number of unselected, consecutive patients ("all comers") with acute BEV, 211 in RCT No. 1 and 154 in RCT No. 2. No comparable studies of similar magnitude have been reported. Unlike other studies, $30 \%$ of the patients were in Child class C with advanced cirrhosis. Both RCTs were community-wide endeavors in which, by prior agreement, $72 \%$ to $82 \%$ of the patients were rapidly referred to UCSD Medical Center from hospitals in four area counties that have a population of 8.5 millions. The entire diagnostic workup, including emergency upper endoscopy, was completed at the bedside in the ICU within less than $8 \mathrm{hr}$ of study entry. Definitive therapy was undertaken within $15 \mathrm{hrs}$ of initial contact with the UCSD staff. EPCS was performed by two senior faculty surgeons with extensive operative experience with portacaval shunt. EEST was performed by boardcertified attending faculty gastroenterologists with a long experience in endoscopic therapy. TIPS was performed by senior attending faculty interventional radiologists with vast experience in TIPS. The type of EPCS was a direct portacaval shunt, side-to-side in almost all patients, end-to-side in a few. Cirrhosis was confirmed by liver biopsy in $100 \%$ of patients. Regular follow-up was accomplished in 100\% of patients and lasted for 10 or more years or until death in RCT No. 1, and for 5 to 10 years or until death in RCT No. 2. No comparable studies with similar long follow-up have been reported.

EPCS by direct anastomosis between the portal vein and IVC permanently controlled BEV in $95 \%$ of patients or more and resulted in long-term survival that was markedly greater than that obtained by TIPS or EST. These results are similar to our recent and past experience with EPCS [8-17]. Moreover, the survival rate is higher than that reported by other investigators who used portosystemic shunts as emergency treatment or to prevent bleeding [26-39]. We believe that several factors were responsible for the consistent results of EPCS that we have obtained over a period of four decades. We reviewed these factors in a recent publication [15]. Briefly, they are: (1) simplification of the diagnostic workup by elimination of unnecessary studies as a routine prior to definitive therapy, which made it possible to accomplish the entire diagnostic workup at the bedside in less than $8 \mathrm{hr}$ without moving the patients out of the ICU, (2) adoption of a specific protocol for an organized system of care before and after EPCS, something that is difficult to accomplish with uniformity in multi-institutional studies. Patients in all four arms of our RCTs were admitted directly to the same ICU where the personnel had specific training and long experience in the care of patients with cirrhosis of the liver, and they were returned to that same ICU postoperatively. Care of all patients in all four arms of the RCTs was supervised by one group of attending physicians throughout the study, (3) rigorous, lifelong program of follow-up in which there was an intensification of efforts to obtain dietary protein control and abstinence from alcohol. Visits to the portal hypertension clinic were scheduled monthly for the first postoperative year and every 3 months thereafter. A dietitian who was employed by the RCT grant and was trained in the care of post-shunt, post-EEST, and post-TIPS patients with cirrhosis was stationed in the clinic to counsel the patient at each visit on the restriction of dietary protein intake to $60 \mathrm{~g} /$ days. Serious efforts were made to enroll all patients who were alcoholics in an alcohol rehabilitation program, such as Alcoholics Anonymous, or a similar program at our own institution. Our patients with portacaval shunts proved to be more receptive to rehabilitation therapy than many alcoholics perhaps because of their almost lethal experience with massive bleeding and EPCS. The frequency of permanent abstinence, as a result, was $85 \%$ in the EPCS group, (4) a low incidence of early and long-term shunt thrombosis. The PCS remained permanently patent in $97.4 \%$ of the patients, which is consistent with our past and recent experience with direct side-to-side PCS [14]. Shunt occlusion represents a serious technical failure of surgical therapy and is usually followed by recurrent BEV and often death. As we documented in our recent publication, high rates of occlusion of various types of shunts have been reported from centers known for experience in the treatment of portal hypertension [15].

A frequent criticism of portal-systemic shunt is the observation or assumption that control of bleeding is achieved at the cost of a high rate of PSE. There is a widespread belief that long-term EST of BEV is associated with a substantially lower rate of PSE than treatment by portal-systemic shunt $[38,39]$. The results of our RCTs are contrary to both of these conclusions. On initial contact before entry in the trial, $19 \%$ of patients in each group had PSE, most likely from gastrointestinal bleeding. A history of PSE was noted in $18 \%$ of the EEST patients and $29 \%$ of the EPCS patients. Chronic, recurrent PSE that required treatment and diminished the quality of life developed in $35 \%$ of patients treated by EEST and 15\% of patients who received EPCS in RCT No. 1 , and in $61 \%$ of the patients treated by TIPS compared to $21 \%$ of those treated by EPCS in RCT No. 2, a significant difference $(\mathrm{p}=0.001)$. Forty percent of the patients who experienced postherapy PSE had PSE pretherapy. This relatively low incidence of PSE in patients with PCS is consistent with our past experience [8-14]. Experience with the patients in this study, and the results in our other studies of EPCS [8-14] demonstrate that a low incidence of PSE is possible when, with the help of rigorous follow-up, patients abstain from alcohol and comply with a diet of moderate protein restriction.

One purpose of the two RCTs that involved 365 randomized patients, and the supplementary analysis of the 1300 unrandomized cirrhotic patients who underwent PCS for BEV was to answer several 
questions about the role of emergency treatment of BEV in subsequent LT. In so doing, it is important to recognize that to date no RCTs have been reported in which the relationship of BEV to LT has been examined prospectively. Since our RCT involved a comparison of EST and TIPS with EPCS, the first question is: what is the role of endoscopic therapy of BEV in patients who might subsequently need LT? Several experienced workers at liver transplantation centers have proposed that EST or Endoscopic Variceal Ligation (EVL) is the first line of treatment for BEV, and that "there are ample data in the literature to support long-term sclerotherapy as the treatment of first choice for bleeding esophageal varices" $[8,9]$. The results of our RCT No. 1 contradict this proposal. EST achieved permanent long-term control of bleeding in only $20 \%$ of unselected consecutive patients. The 5 -year survival rate of patients treated by EST was only $21 \%$, and median survival was less than 3 years, even with rescue PCS. The reasons why LT was not considered in more patients in the EST group are that death from recurrent bleeding in $44 \%$ of patients, frequent PSE often due to noncompliance as well as to recurrent bleeding, and social issues such as continued alcoholism, drug abuse and non-USA residence status obviated considerations of LT. The results of TIPS in RCT No. 2 were not much different.

The second question is: how often following an episode of acute BEV in patients with advanced cirrhosis is LT required? The results of our study indicate that LT is seldom required following control of bleeding by PCS. In our two RCTs, only $6 \%$ of the patients were referred for LT, only $3 \%$ were approved for LT, and only 8 patients $(2 \%)$ underwent LT. Of our 1300 unrandomized patients with PCS, only $3.8 \%$ of the patients were referred for LT, and only 19 patients (1.5\%) underwent LT. Furthermore and most important, if recurrent BEV is prevented, as was true in $100 \%$ of the PCS patients, both randomized and unrandomized, prolonged survival occurs, equal to or better than survival following LT. 30 -days and 5-years survival rates were $87 \%$ and 73\%, respectively, following EPCS in 105 patients in RCT No. 1, $83 \%$ and $67 \%$, respectively, following EPCS in 600 unrandomized patients, and $98 \%$ and $71 \%$, respectively, following elective PCS in 700 unrandomized patients. Survival rates following LT during the period 1988 to 2001 in 46,940 patients reported by the United Network for Organ Sharing (UNOS) were $74.5 \%$ at 1 year, $67.4 \%$ at 3 years, and $62.7 \%$ at 5 years [41]. Clearly, the 5 -years survival rate following PCS $(73 \%, 67 \%$, and $71 \%)$ was higher than that of the UNOS LT survival rate $(62.7 \%)$. It is noteworthy that close to one-half of the patients in the RCT (46\%) were alive 15 years after EPCS.

The third question is: should transplant surgeons avoid portacaval shunts or surgical decompressive procedures that involve the hepatic hilum in potential LT candidates? There are numerous statements in the transplantation literature urging avoidance of PCS and strong recommendations to that effect from some recognized transplantation centers $[5,6,42-45]$. However, there are no valid, representative data from prospective studies, randomized or unrandomized, that support such a proposal. Our studies indicate that PCS is the most effective treatment of $\mathrm{BEV}$, invariably prevents recurrence of bleeding, and achieves long-term survival so that the vast majority of patients do not require $\mathrm{LT}$.

The fourth question is: do portosystemic shunts and, particularly, portacaval shunt, affect the outcome of LT? Our RCTs in which only 8 of the 365 patients underwent LT do not provide sufficient data to answer this question. However, the survival rate of the 19 unrandomized patients who underwent LT was $89 \%$ after 30 days and $53 \%$ after 5 years, which was not significantly different from the survival rates for LT reported by UNOS. PCS did not increase the mortality rate or complications of subsequent LT. Added to the data from our study are at least 10 reports of retrospective studies in the literature that concluded that portosystemic shunts do not affect the results of subsequent LT [5-7,43-51]. That conclusion has not been contradicted by reported studies.

Liver transplantation plays a central role in the treatment of cirrhosis of the liver. Nevertheless, there are a number of limitations on the use of this important therapeutic measure as a cure for cirrhosis [52]. The most important limitation is unavailability of donor livers. In year 2008 there were 17,167 candidates on the waiting list for LT at the beginning of the year and 16,538 on the waiting list at the end of the year (based on OPTN data as of /31/09). 6,319 candidates, or $37 \%$, received LT. 2,456 patients died or deteriorated while awaiting LT. The median waiting time for LT in 2002 was 974 days and, although the wait was shorter in 2005, it was still 321 days [41]. Furthermore, numerous patients referred for LT have been disqualified for various reasons. Of the patients referred for LT evaluation, $46 \%$ in the RCTs and $62 \%$ in the unrandomized series were turned down for valid reasons including continued alcoholism, drug abuse, and non-USA resident status. Additionally, the costs of LT are substantial. Direct costs of care, as reflected by charges for patients who underwent EPCS in our RCT including costs of lifelong follow-up, averaged a total of $\$ 150,400$ or $\$ 39,400$ per year. In contrast, the mean cost of LT for cirrhosis in adult patients in 2008, including 180 days of post-transplant care, averaged $\$ 523,400$, which is more than 3 times the lifelong average cost of EPCS [41]. Finally, third-party insurance coverage for emergency treatment of BEV such as EPCS has been readily obtainable, while coverage for LT is sometimes difficult to obtain. At one of our institutions, of 182 patients referred for LT from January through July, 2009, 11\% were denied LT because of insurance issues.

In conclusion, in these RCTs of emergency treatment of acute BEV in 365 patients with cirrhosis, supplemented by analyses of 1300 unrandomized patients treated by portacaval shunt, prospective data indicate clearly that BEV by itself should not be considered an indication for LT. Moreover, the data show that PCS should be considered a firstline treatment for BEV since it promptly and permanently stopped variceal bleeding, was accomplished with a relatively low (15\%) incidence of subsequent PSE, and produced long-term survival rates equal or superior to those produced by LT. Furthermore, PCS did not increase the mortality rate or complications in the small number of patients who ultimately required LT, a finding that was confirmed by at least 10 retrospective studies reported in the literature. The importance of these results is underscored by the severe shortage of donor organs, the much higher cost of LT compared to PCS, and the substantial difficulties in obtaining insurance coverage for LT in some patients in the population that develops BEV due to cirrhosis.

\section{Acknowledgements}

We thank the many residents in the Department of Surgery, the Department of Radiology, and the Department of Medicine who played a major role in the care of patients in this study. We are particularly grateful to Dr. Karim Valji and his colleagues of the Department of Radiology who played an important role in the conduct of the radiologic procedures such as TIPS. We thank Professors Harold O. Conn of Yale University; Haile T. Debas and Alexander R. Margulis of the University of California, San Francisco; and Peter B. Gregory of Stanford University, who served voluntarily as an External Advisory, Data Safety and Monitoring Committee. We thank the many physicians practicing in the counties of San Diego, Imperial, Orange, and Riverside who helped with patient recruitment referral, and long-term follow-up. This work was supported in part by Health Resources and Services Administration contract 234-2005-370011C. The content is the responsibility of the authors alone and does not necessarily reflect the views or policies of the Department of Health and Human Services nor does mention of trade names, commercial products, or organizations imply endorsement by the U.S. Government. 
Citation: Orloff MJ (2013) Liver Transplantation during Fifty-Three Years' Experience with Randomized Controlled Trials of Emergency Portacaval Shunt for Bleeding Esophageal Varices in Cirrhosis. Surgery Curr Res 3: 140. doi:10.4172/2161-1076.1000140

Page 12 of 13

\section{Conflict of interest}

There was no conflict of interest relevant to this article on the part of any of the authors.

\section{Grant \& Funding}

Supported by grants 1R01 DK41920 and AM17103 from the National Institutes of Health and grants from the Surgical Education and Research Foundation [501(c) (3)], clinicaltrials.gov \#NCT00690027 and \#NCT00734227.

\section{References}

1. Graham DY, Smith JL (1981) The course of patients after variceal hemorrhage. Gastroenterology 80: 800-809.

2. Smith JL, Graham DY (1982) Variceal hemorrhage: a critical evaluation of survival analysis. Gastroenterology 82: 968-973.

3. Orloff MJ, Duguay LR, Kosta LD (1977) Criteria for selection of patients for emergency portacaval shunt. Am J Surg 134: 146-152.

4. Mikkelsen WP (1974) Therapeutic portacaval shunt. Preliminary data on controlled trial and morbid effects of acute hyaline necrosis. Arch Surg 108 302-305

5. Iwatsuki S, Starzl TE, Todo S, Gordon RD, Tzakis AG, et al. (1988) Liver transplantation in the treatment of bleeding esophageal varices. Surgery 104 697-705

6. Reyes J, Iwatsuki S (1992) Current management of portal hypertension with liver transplantation. Adv Surg 25: 189-208.

7. Bismuth H, Adam R, Mathur S, Sherlock D (1990) Options for elective treatmen of portal hypertension in cirrhotic patients in the transplantation era. Am J Surg 160: 105-110.

8. Orloff MJ (1967) Emergency portacaval shunt: a comparative study of shunt, varix ligation and nonsurgical treatment of bleeding esophageal varices in unselected patients with cirrhosis. Ann Surg 166: 456-478.

9. Orloff MJ, Chandler JG, Charters AC, Condon JK, Grambort DE, et al. (1974) Emergency portacaval shunt treat?ment for bleeding esophageal varices. Prospective study in unselected patients with alcoholic cirrhosis Arch Surg 108: 293-299.

10. Orloff MJ, Charters AC 3rd, Chandler JG, Condon JK, Grambort DE, et al. (1975) Portacaval shunt as emergency procedure in unselected patients with alcoholic cirrhosis. Surg Gynecol Obstet 141: 59-68.

11. Orloff MJ, Bell RH Jr, Hyde PV, Skivolocki WP (1980) Long-term results of emergency portacaval shunt for bleeding esophageal varices in unselected patients with alcoholic cirrhosis. Ann Surg 192: 325-340.

12. Orloff MJ, Orloff MS, Rambotti M, Girard B (1992) Is portal-systemic shun worthwhile in Child's class $C$ cirrhosis? Long-term results of emergency shun in 94 patients with bleeding varices. Ann Surg 216: 256-266.

13. Orloff MJ, Bell RH Jr, Orloff MS, Hardison WG, Greenburg AG (1994) Prospective randomized trial of emergency portacaval shunt and emergency medical therapy in unselected cirrhotic patients with bleeding varices. Hepatology 20: 863-872.

14. Orloff MJ, Orloff MS, Orloff SL, Rambotti M, Girard B (1995) Three decades of experience with emergency portacaval shunt for acutely bleeding esophageal varices in 400 unselected patients with cirrhosis of the liver. J Am Coll Surg 180: $257-272$.

15. Orloff MJ, Isenberg JI, Wheeler HO, Haynes KS, Jinich-Brook H, et al. (2009) Randomized trial of emergency endoscopic sclerotherapy versus emergency portacaval shunt for acutely bleeding esophageal varices in cirrhosis. J Amer Coll Surg 209: 25-40.

16. Orloff MJ, Isenberg JI, Wheeler HO, Haynes KS, Jinich-Brook H, et al. (2010) Direct costs of care in a randomized controlled trial of endoscopic sclerotherapy versus emergency portacaval shunt treatment of acutely bleeding esophageal varices in cirrhosis - part 3. J Gastrointestinal Surg 14: 1782-1795.

17. Orloff MJ, Vaida F, Haynes KS, Hye RJ, Isenberg JI, et al. (2012) Randomized controlled trial of emergency transjugular intrahepatic portosystemic shunt versus emergency portacaval shunt treatment of acute bleeding esophageal varices in cirrhosis. J Gastrointest Surg 16: 2094-2111.

18. Orloff MJ, Isenberg JI, Wheeler HO, Haynes KS, Jinich-Brook H, et al. (2010) Liver transplantation in a randomized controlled trial of emergency treatment of acutely bleeding esophageal varices in cirrhosis. Transplantation Proc 42 4101-4108.

19. Moher D, Schulz KF, Altman D (2001) The CONSORT statement revised recommendations for improving the quality of reports of parallel-group randomized trials. JAMA 285: 1987-1991.

20. Altman DG, Schulz KF, Moher D, Egger M, Davidoff F, et al. (2001) The revised CONSORT statement for reporting randomized trials: explanation and elaboration. Ann Intern Med 134: 663-694.

21. Schulz KF, Altman DG, Moher D, for the Group CONSORT 2010 statement: Updated guidelines for reporting parallel group randomization trials 340:C332.

22. Campbell DP, Parker DE, Anagnostopoulos CE (1973) Survival prediction in portacaval shunts: a computerized statistical analysis. Am J Surg 126: 748-751.

23. Child III, CG, Turcotte JG (1964) Surgery and portal hypertension. In: The Live and Portal Hypertension. Edited by CG Child III. W.B. Saunders, Philadelphia, pp. 1-85.

24. Orloff MJ, Isenberg JI, Wheeler HO, Haynes KS, Jinich-Brook H, et al. (2009) Portal-systemic encephalopathy in a randomized controlled trial of endoscopic sclerotherapy versus emergency portacaval shunt treatment of acutely bleeding esophageal varices in cirrhosis. Ann Surg 250: 598-610.

25. Orloff MD, Vaida F, Haynes KS, Hye RJ, Isenerg JI, et al. (2013) Portal-systemic encephalopathy in a randomized controlled trial of emergency transjugular intrahepatic portosystemic shunt versus emergency portacaval shunt treatment of acute bleeding esophageal varices in cirrhosis. Am J Surg.

26. Korula J, Yelin A, Yamada S, Weiner J, Cohen H, et al. (1987) A prospective randomized controlled comparison of chronic endoscopic variceal sclerotherapy and portal-systemic shunt for variceal hemorrhage in Child's class A cirrhotics: a preliminary report. Gastroenterology 92:1745.

27. Planas R, Boix J, Broggi M, Cabré E, Gomes-Vieira MC, et al. (1991) Portacava shunt versus endoscopic sclerotherapy in the elective treatment of variceal hemorrhage. Gastroenterology 100: 1078-1086.

28. Isaksson B, Jeppsson B, Bengtsson F, Hannesson P, Herlin P, et al. (1995) Mesocaval shunt or repeated sclerotherapy: effects on rebleeding and encephalopathy--a randomized trial. Surgery 117: 498-504.

29. Henderson JM, Kutner MH, Millikan WJ, Galambos JT, Riepe SP, et al. (2009) Endoscopic variceal sclerosis compared with distal splenorenal shunt to prevent recurrent variceal bleeding in cirrhosis, a prospective randomized trial. Ann Intern Med 112:262-269.

30. Rikkers LF, Burnett DA, Volentine GD, Buchi KN, Cormier RA (1987) Shun surgery versus endoscopic sclerotherapy for long-term treatment of variceal bleeding. Early results of a randomized trial. Ann Surg 206: 261-271.

31. Rikkers LF, Jin G, Burnett DA, Buchi KN, Cormier RA (1993) Shunt surgery versus endoscopic sclerotherapy for variceal hemorrhage: late results of a randomized trial. Am J Surg 165: 27-32.

32. Terés J, Bordas JM, Bravo D, Visa J, Grande L, et al. (1987) Sclerotherapy vs. distal splenorenal shunt in the elective treatment of variceal hemorrhage: a randomized controlled trial. Hepatology 7: 430-436.

33. Spina GP, Santambrogio R, Opocher E, Cosentino F, Zambelli A, et al. (1990) Distal splenorenal shunt versus endoscopic sclerotherapy in the prevention of variceal rebleeding. First stage of a randomized, controlled trial. Ann Surg 211: 178-186.

34. Gralnek IM, Jensen DM, Kovacs TO, Jutabha R, Machicado GA, et al. (1999) The economic impact of esophageal variceal hemorrhage: cost-effectiveness implications of endoscopic therapy. Hepatology 29: 44-50.

35. Villeneuve JP, Pomier-Layrargues G, Duguay L, Lapointe R, Tanguay S, et al. (1987) Emergency portacaval shunt for variceal hemorrhage. A prospective study. Ann Surg 206: 48-52.

36. Soutter DI, Langer B, Taylor BR, Greig P (1989) Emergency portasystemic shunting in cirrhotics with bleeding varices--a comparison of portacaval and mesocaval shunts. HPB Surg 1: 107-116.

37. Spina GP, Santambrogio R, Opocher E, Gagliano G, Cucchiaro G, et al (1990) Emergency portosystemic shunt in patients with variceal bleeding. Surg Gynecol Obstet 171: 456-464.

38. Garcia N Jr, Sanyal AJ (2001) Portal hypertension. Clin Liver Dis 5:1-26.

39. Klempnaue J, Schrem H (2001) Review: surgical shunts and encephalopathy Metab Brain Dis 16: 21-25. 
Citation: Orloff MJ (2013) Liver Transplantation during Fifty-Three Years' Experience with Randomized Controlled Trials of Emergency Portacaval Shunt for Bleeding Esophageal Varices in Cirrhosis. Surgery Curr Res 3: 140. doi:10.4172/2161-1076.1000140

Page 13 of 13

40. Hauboldt RH, Hanson SG, Bernstein GR (2008) Organ and tissue transplant cost estimates and discussion. Milliman Research Report 1-17.

41. Sorbi D, Gostout CJ, Peura D, Johnson D, Lanza F, et al. (2003) An assessment of the management of acute bleeding varices: a multicenter prospective member-based study. Am J Gastroenterol 98: 2424-2434.

42. Brems JJ, Hiatt JR, Klein AS, Millis JM, Colonna JO, et al. (1989) Effect of a prior portasystemic shunt on subsequent liver transplantation. Ann Surg 209: $51-56$.

43. Jenkins RL, Gedaly R, Pomposelli JJ, Pomfret EA, Gordon F, et al. (1999) Distal splenorenal shunt: role, indications, and utility in the era of liver transplantation. Arch Surg 134: 416-420.

44. Dell'Era A, Grande L, Barros-Schelotto P, Turnes J, Fuster J, et al. (2005) Impact of prior portosystemic shunt procedures on outcome of liver transplantation. Surgery 137: 620-625.

45. Mazzaferro V, Todo S, Tzakis AG, Stieber AC, Makowka L, et al. (1990) Liver transplantation in patients with previous portasystemic shunt. Am J Surg 160: 111-116.
46. Langnas AN, Marujo WC, Stratta RJ, Donovan JP, Sorrell MF, et al. (1992) Influence of a prior porta-systemic shunt on outcome after liver transplantation. Am J Gastroenterol 87: 714-718.

47. Turrion VS, Mora NP, Cofer JB, Solomon H, Morris CA, et al. (1991) Retrospective evaluation of liver transplantation for cirrhosis: a comparative study of 100 patients with or without previous porto-systemic shunt. Transplant Proc 23: 1570-1571.

48. AbouJaoude MM, Grant DR, Ghent CN, Mimeault RE, Wall WJ (1991) Effect of portasystemic shunts on subsequent transplantation of the liver. Surg Gynecol Obstet 172: 215-219.

49. Rubio Gonzalez EE, Moreno Planas JM, Jimenez Garrido MC, Lucena de la Poza JL, Martínez Arrieta F, et al. (2005) Results of liver transplantation in patients with previous portosystemic shunts. Transplant Proc 37: 1491-1492.

50. Ho KS, Lashner BA, Emond JC, Baker AL (1993) Prior esophageal variceal bleeding does not adversely affect survival after orthotopic liver transplantation. Hepatology 18: 66-72.

51. Anantharaju A, Van Thiel DH (2003) Liver transplantation for alcoholic liver disease. Alcohol Res Health 27: 257-268. 